\title{
On the foundations of constructive mathematics - especially in relation to the theory of continuous functions
}

\author{
Frank Waaldijk *
}

July 6, 2003

\begin{abstract}
We discuss the foundations of constructive mathematics, including recursive mathematics and intuitionism, in relation to classical mathematics. There are connections with the foundations of physics, due to the way in which the different branches of mathematics reflect reality. Many different axioms and their interrelationship are discussed. We show that there is a fundamental problem in BISH (Bishop's school of constructive mathematics) with regard to its current definition of 'continuous function'. This problem is closely related to the definition in BISH of 'locally compact'.

Possible approaches to this problem are discussed. Topology seems to be a key to understanding many issues. We offer several new simplifying axioms, which can form bridges between the various branches of constructive mathematics and classical mathematics ('reuniting the antipodes'). We give a simplification of basic intuitionistic theory, especially with regard to so-called 'bar induction'. We then plead for a limited number of axiomatic systems, which differentiate between the various branches of mathematics. Finally, in the appendix we offer BISH an elegant topological definition of 'locally compact', which unlike the current definition is equivalent to the usual classical and/or intuitionistic definition in classical and intuitionistic mathematics respectively.
\end{abstract}

*currently employed at Royal Haskoning, Nijmegen, The Netherlands. Email at home: goudmos50@hetnet.nl 


\section{INTRODUCTION}

1.1 This paper is concerned with the foundations of constructive mathematics, as the title suggests. There are connections with the foundations of physics, due to the way in which the different branches of mathematics reflect reality. As an example, our investigations lead to a physical experiment which might give statistical evidence whether the physical world is 'deterministic' or not. This old debate is put in a clear-cut mathematical perspective which we feel deserves attention, even though the experiment most likely will fail to settle the debate.

The main focus of the paper is however of a mathematical nature. The paper discusses many different axioms and their interrelationship. In the introduction we will already use their abbreviations such as FT, MP, $\mathbf{A C}_{11}$, etc. No worry however: all these abbreviations will be treated in detail in the rest of the paper. Finally some recommendations are made for a constructive framework, which should form the real benefit of this paper. We start with a brief historical outline.

1.2 In the beginning of the twentieth century Brouwer (in [Brouwer1907]) put forward a sharp foundational critique of classical mathematics (CLASS). Having made a name for himself in the new field of topology through a number of impressive results, Brouwer turned to building a new mathematics: intuitionism (INT). Though at this time heard by many, his views were not largely accepted, not in the least because his critique seemingly left most of classical mathematics in ashes. The outcome of Brouwer's dispute with Hilbert illustrates that even mathematics is no objective, but a subjective and also very social affair. Most mathematicians continue to follow Hilbert's classical views, but without a proper understanding of Brouwer's critique. Classical mathematics is mainstream, and taught for instance in high school. Intuitionism is an oddity, seen mostly as useful in logic and informatics, and hardly anyone encounters it.

Yet a number of well-known classical mathematicians supported Brouwer throughout the twentieth century. For this paper we especially name Kleene, who became famous for his work on recursion theory, and Bishop, a well-known analyst. Kleene wrote a book together with Vesley ([Kleene\&Vesley1965]) called 'The Foundations of Intuitionistic Mathematics - especially in relation to the theory of recursive functions'. In this book Kleene presents Brouwer's insights in a clear and straightforward way, and axiomatizes them. Then he shows that Brouwer's framework is consistent. For this Kleene uses the theory of recursive functions and a notion called 'realizability'. In passing, Kleene also shows that Brouwer's fan theorem FT is inconsistent with the assumption that all sequences of natural numbers are given by a recursive rule. This assumption is called 'Church's thesis' (CT). CT is adopted together with Brouwer's intuitionistic logic by a group of mainly Russian mathematicians (notably Markov), to form a branch of constructive mathematics known as Russ.

In logic and later on informatics the impact of Brouwer's intuitionism was profoundly felt. But notwithstanding Kleene's and other people's work, Brouwer's impact on mainstream mathematics seemed to dwindle. Then in [Bishop1967] Bishop introduced what we will call BISH: a variety of constructive mathematics which adopts much of Brouwer's intuitionistic views, but at the same time rejects Brouwer's most striking insights such as the fan theorem $\mathbf{F T}$ and the axiom of continuous choice $\mathbf{A C}_{11}$.

Bishop wanted to use largely the same terminology as classical mathematics, with the same primarily mathematical focus. In this way more mathematicians could understand constructive mathematics and 
be attracted to it. Bishop viewed the axioms and terminology of intuitionism as mystic (notwithstanding the clarifications offered in [Kleene\&Vesley1965], which although phrased in 'logical language' form a solid axiomatic foundation for INT $)^{1}$. He preferred to first develop in a constructive way as much of classical analysis as possible, before thinking things through foundationally. Out of his work, Richman and Bridges developed a view of BISH as a mathematics, the methods and results of which are acceptable in CLASS, INT and RUSS alike. Hereby they disregard the philosophical differences, which give very different interpretations of the objects and the universe involved.

Bishop's approach was quite successful, and attracted a respectable number of mathematicians. This gave new life to constructive mathematics. We will show in this paper however that BISH, contrary to its claim, does not take up a neutral position with respect to the antipodes CLASS, INT and RUSS. Also we are led to investigate several mathematical and meta-mathematical issues which impact on the foundations of INT, RUSS, CLASS, and BISH. One conclusion that we like to draw right here is that topology (Brouwer's specialty) provides a fundamental key for understanding these issues. Topology has not received much attention yet in constructive mathematics, although powerful topological tools from classical mathematics can also be developed in BISH and INT. ${ }^{2}$

1.3 Bishop maintained that in the context of analysis one could do without FT and axioms of continuity by restricting one's attention to continuous functions known to be uniformly continuous on compact subspaces of their domain. Only these functions deserved his predicate 'continuous', other functions if existent did not merit much attention. It therefore seems that intuitively at least Bishop agreed with Brouwer's analysis which led Brouwer to put forward FT. One can show that the role of this axiom is nothing more than to bring about the same restriction on continuous functions.

For in this paper we show that the existence of any class of 'continuous functions' satisfying the above property 'uniformly continuous on compact subspaces' and a rather minimal list of other conditions is equivalent within BISH to the fan theorem FT. This throws a fundamental light on both BISH and FT, which has not received the attention it deserves in the constructive literature. The main theorem in this respect is:

THEOREM: Within BISH the following statements are equivalent:

(1) The fan theorem FT.

(2) There exists a class of real-valued functions called 'kontinuous' functions such that:

(a) If $f$ is a uniformly continuous real-valued function defined on $[0,1]$, then $f$ is kontinuous.

(b) If $f$ and $g$ are kontinuous functions such that $\operatorname{Ran}(f) \subseteq \operatorname{Dom}(g)$, then the composition $g \circ f$ is kontinuous.

(c) If $f$ is a kontinuous function defined on $[0,1]$, then $f$ is uniformly continuous.

(d) The function $x \longmapsto \frac{1}{x}$, defined on $\mathbb{R}^{+}$, is kontinuous.

From this theorem we derive a result concerning the two different definitions of 'continuous function' which are currently being used in BISH, namely the definition in [Bishop1967] (repeated in [Bishop\&Bridges1985])

\footnotetext{
${ }^{1}$ Bishop did mention [Kleene\&Vesley1965] in his paper 'Schizophrenia in Contemporary Mathematics', [Bishop1985], as well as in its list of references.

${ }^{2}$ See [Waaldijk1996].
} 
and the definition in [Bridges1979]. The latter is an attempt to repair the [Bishop1967] definition. ${ }^{3}$ To formulate this result let us call the first definition 'continuous ${ }^{\mathrm{BIS}}$ ' and the second definition 'continuous ${ }^{\mathrm{BRI}}$ '.

COROLLARY: Within BISH the following three statements are equivalent:

(1) The fan theorem FT.

(2) If $f$ is a continuous ${ }^{\text {BIs }}$ function from $[0,1]$ to $\mathbb{R}^{+}$, then the composition $x \longmapsto \frac{1}{f(x)}$ of $f$ with the continuous ${ }^{\mathrm{BIS}}$ function $x \longmapsto \frac{1}{x}$ is continuous ${ }^{\mathrm{BIS}}$.

(3) The function $x \longmapsto \frac{1}{x}$, defined on $\left(\mathbb{R}^{+}, d_{\mathbb{R}}\right)$, is continuous ${ }^{\mathrm{BRI}}$.

REMARK: The following statement (more general than (2) above) therefore implies FT:

(2') If $f$ and $g$ are real-valued continuous ${ }^{\text {BIs }}$ functions such that $\operatorname{Ran}(f) \subseteq \operatorname{Dom}(g)$, then the composition $g \circ f$ is continuous ${ }^{\mathrm{BIS}}$.

However, we do not see how to prove (2') using only FT. There is a proof of (2') using FT and a weak, classically true, version of $\mathbf{A C}_{11}$ (see 8.8).

1.4 Further on we study theorem 1.3 and its relation with the foundations of BISH. We look at the consequences of omitting one or more conditions on 'continuous function' from the list (2)(a) through $(2)(d)$ in the above theorem.

1.5 As a side product of our study, we obtain within the framework of RUss a counterexample to FT which is in essence the same as the counterexample in [Kleene\&Vesley1965], but with a different flavour: we present a recursive element of the Hilbert cube which is recursively apart from every recursive binary element of the Hilbert cube (a binary element has a binary expansion in each coordinate). Recursive mathematics (RUSS) plays an important role in our discussion. RUSS takes the viewpoint that any infinite sequence must be given by a finite algorithm (recursive rule) which produces the sequence step by step ${ }^{4}$. If applied to the physical world, the viewpoint of RUSS in the author's eyes corresponds to the philosophical standpoint of our world being deterministic. We will elucidate this in section 7 .

Early in his intuitionistic career Brouwer maintained similar ideas, but later on he dropped the algorithmic requirement and stated that infinite sequences can be produced step by step, without any rule for and any prior knowledge of what the next steps might bring. Brouwer based his step-by-step creative sequences on the human intuition of infinite time. Of course one can restrict oneself beforehand to follow a recursive rule, but according to Brouwer this is no necessity. Therefore in Brouwer's universe one has in the strongest sense possible what we will call 'incomplete information'5. If applied to the

\footnotetext{
${ }^{3}$ These definitions are not equivalent, yet so far in constructive papers no explicit reference is made to this duality (can results from [Bridges1979] be used when the definitions come from [Bishop\&Bridges1985]?).

${ }^{4}$ Thinking things through, the author does not see how to convince himself that a certain sequence has infinite potential, unless we produce precisely such a finite algorithm and show that it will produce a result for every $n \in \mathbb{N}$. What guarantee do we have that other sequences, for instance from reality, are potentially infinite? What if this world comes to an end? Of course then any algorithm would also stop, but at least one has the feeling that the algorithms as developed by Church and Turing will be the same in any possible other world. Like an encapsulated virus an algorithmic infinite sequence can be turned on at any time, in any situation, to produce the same outcome.

${ }^{5}$ However, one also has incomplete information in RUSS, since it is impossible to algorithmically determine for an arbitrary algorithm whether it will produce in fact an infinite sequence, or whether it fails on a given input.
} 
physical world, the viewpoint of both INT and CLASS in the author's eyes implies that our world is NOT deterministic, see section 7 .

The failure of FT in RUss can be ascribed to the extra information that one has about elements of the mathematical universe $\mathbb{N}^{\mathbb{N}}$, namely that every such element is produced by a finite algorithm. In RUSS each sequence is even produced by a known finite algorithm. However, reality comes to us in a different way. Many natural phenomena change with time, and therefore present us with sequences of numbers for which we have a priori no algorithm. This does not prove that such an algorithm ultimately doesn't exist. Who knows what the physicists or the theologians may come up with? But so far in most cases we cannot pinpoint any such algorithm.

So we have basically two possible ways to model our real world. The first way is to assume that it is non-deterministic, and then both INT and CLASS are remaining. The difference between these then is the difference between a world of incomplete information (INT) and a world of complete information (CLASS). Interpreted in this way it is clear that CLASS leads to an unrealistic model, since in real life we deal with many situations where we do not have complete information. So the principle of the excluded middle (PEM, 'For all A: A is true or A is false') often doesn't apply since we have no method to tell which of the extremes is true. Brouwer very sharply demonstrated this mathematically, and also identified the cause of the problems: the intuition behind PEM comes from finite decidable situations, but this intuition cannot be transferred outside this context.

The second way to model our real world is to assume that it is deterministic. Then both RUSS and classical recursion theory remain applicable. Classical recursion theory is again a world of complete information. But RUSS is in between 'complete information' and 'incomplete information'. It would be worthwile to explore the consequences of a deterministic world with incomplete information (since under the assumption of determinacy in the author's eyes this comes closest to real life). That is a world in which each infinite sequence is given by an algorithm, which in most cases is completely unknown. We can model such a world by introducing two players, where player I picks algorithms and hands out the computed values of these algorithms to player II, one at a time. Sometimes player I discloses (partial) information about the algorithms themselves. Player II can of course construct her or his own algorithms, but still is confronted with recursive elements of player I about which she/he has incomplete information. One is tempted to think that in such a world intuitionistic principles might hold. We believe $\mathbf{C P}$ will be consistent. But it is not difficult to show that FT remains invalid in such a world.

There is a surprising conclusion to this line of thought. It is possible to conduct a physical experiment which might give statistical evidence whether the real world is deterministic or not (in the interpretation of determinism as preferred by the author, see section 7). We believe that the actual carrying out of this experiment can be of relevance to both mathematical foundations as to physics and philosophy in general. The experiment is based on the way in which FT either fails or holds, given the determinacy or non-determinacy assumption.

1.6 Our investigations lead us to consider the possibilities for 'reuniting the antipodes' ${ }^{6}$. The antipodes here being classical mathematics (CLASS) and intuitionism (INT). From a purely axiomatic point of view, they have far more in common than the current scope of BISH, and they are much closer to each other than to Russ. As stated, they also share the non-determinacy of the universe as a philosophical insight. It therefore seems worthwile to explore the 'formal' common ground of classical and intuitionistic

\footnotetext{
${ }^{6}$ We gladly adapt the terminology of Peter Schuster to this setting.
} 
mathematics. If systematically developed, many intuitionistic results would be seen to hold classically as well, and thus would offer a way to develop a strong constructive theory which is still consistent with the rest of classical mathematics. Such a constructive theory can form a conceptual framework for applied mathematics, and information technology. These sciences now use an ad-hoc approach to reality, since the classical framework is inadequate. This ad-hoc approach leads to inefficiency in methods used and in research conducted. Bishop's motivation to develop BISH was similar. But INT is stronger, and comes closer to CLASS.

The intuitionist can on the other hand more easily use the richness of ideas already present in classical mathematics, if classical mathematics were to be systematically developed along the common grounds before the unconstructive elements are brought in. ${ }^{7}$

One can also investigate the 'formal' common ground of all three varieties CLASS, INT and RUSS. This common ground is much less than that of CLASS and INT, but still is larger than what is offered in BISH. We will indicate several nice theorems belonging to the common ground of all three varieties. 8

One of the conclusions is that basic topology is too often neglected in constructivism. Many approaches and results in the constructive literature can be obtained in a simpler way with the help of basic topology ${ }^{9}$. Especially from the standpoint of topology, the current BISH definition of 'continuous function' merits revision, and the same holds for the current BISH-definition of 'locally compact'.

Finally the paper offers some simplification of basic intuitionistic theory regarding bars and bar induction. A further strengthening of intuitionism can be obtained by adopting Markov's Principle MP (true in CLASS and RUSS). The author believes that there is very strong intuitive support for MP, and intuitionism will benefit from the simplifications that MP brings about.

\section{Basic Axioms and Definitions}

2.1 In this section we present some basic axioms described in the literature, pertaining to constructive mathematics in particular (INT and RUSS). To define the relevant intuitionistic axioms of (continuous) choice we need a number of straightforward definitions.

DEFINITION: Let $\sigma_{\omega}$ denote the universal spread of all infinite sequences of natural numbers $\left(\sigma_{\omega}=\mathbb{N}^{\mathbb{N}}\right)$. On $\sigma_{\omega}$ the natural metric $d_{\omega}$ is defined by putting, for $\alpha$ and $\beta$ in $\sigma_{\omega}: d_{\omega}(\alpha, \beta)=\sup \left(\left\{2^{-n} \mid \alpha(n) \neq\right.\right.$ $\beta(n) \mid n \in \mathbb{N}\}$. Write $\bar{\sigma}_{\omega}$ for the set of finite sequences of natural numbers (often written like this:

\footnotetext{
${ }^{7}$ However, much of classical mathematics will remain an exercise in charting the consequences of the unrealistic principle of the excluded middle. An intuitionist will be hard put to understand that this is a worthwile pursuit.

${ }^{8}$ Also intuitionism and recursive mathematics share a noteworthy topological insight: in a complete metric space the so-called 'apartness topology' coincides with the metric topology; which explains the metric continuity of everywheredefined functions in a more fundamental way than the usual approach. For Russ, this insight relies partly on Markov's Principle MP which is classically true. We will plead for MP also in the context of INT.

${ }^{9}$ For many results on complete metric spaces $(X, d)$ it suffices to prove the result for $\left(\sigma_{\omega}, d_{\omega}\right)$, and then consider a continuous surjection from $\left(\sigma_{\omega}, d_{\omega}\right)$ to $(X, d)$, 'pulling back' the topology. The same holds, mutatis mutandis, for compact spaces and $\left(\sigma_{2}, d_{\omega}\right)$. Also consider so-called 'partitions of unity' (see [Waaldijk1996]). With the help of this tool one proves the Dugundji extension theorem, rather than the weaker Tietze extension theorem. Also see the previous footnote. There is a large list of similar simplifications.
} 
$\left.\bar{\sigma}_{\omega}=\mathbb{N}^{*}\right)$. For $\alpha$ in $\sigma_{\omega}$ we write $\bar{\alpha}(n)$ for the finite sequence $\alpha(0), \ldots, \alpha(n-1)$ formed by the first $n$ values of $\alpha$. Then $\bar{\alpha}(n) \in \overline{\bar{\sigma}}_{\omega}$, and vice versa $\overline{\bar{\sigma}}_{\omega}=\left\{\bar{\alpha}(n) \mid \alpha \in \sigma_{\omega}, n \in \mathbb{N}\right\}$. A subset $B$ of $\overline{\bar{\sigma}}_{\omega}$ is called decidable iff $\forall a \in \overline{\bar{\sigma}}_{\omega}[a \in B \vee a \notin B]$. A subset $B$ of $\overline{\bar{\sigma}}_{\omega}$ is a bar on a subset $A$ of $\sigma_{\omega}$ iff $\forall \alpha \in A \exists n \in \mathbb{N}[\bar{\alpha}(n) \in B]$. Notice that a bar on $A$ is the same as an open cover of $A$ consisting of basic open sets (in the product topology).

Now let $a$ be in $\overline{\bar{\sigma}}_{\omega}$, in other words $a$ is a finite sequence of natural numbers. Then we write $\lg (a)$ for the length of this finite sequence. So if $a=a_{0}, \ldots, a_{n-1}$ then $l g(a)=n$. There is a sequence of length 0 , namely the empty sequence denoted by $\varangle \triangleright$. For $i<\lg (a)$ we then write $a_{i}$ for the $i^{\text {th }}$ element of this finite sequence. If $a=a_{0}, a_{1}, \ldots, a_{l g(a)-1}$ and $b=b_{0}, b_{1}, \ldots, b_{l g(b)-1}$ are in $\bar{\sigma}_{\omega}$ then we write $a \star b$ for the concatenation $a_{0}, a_{1}, \ldots, a_{l g(a)-1}, b_{0}, b_{1}, \ldots, b_{l g(b)-1}$ of $a$ and $b$. We write $a \sqsubseteq b$ iff there is a $c$ in $\overline{\bar{\sigma}}_{\omega}$ such that $b=a \star c$, and we write $a \sqsubset b$ iff in addition $\lg (b)>\lg (a)$.

Likewise for $\alpha$ in $\sigma_{\omega}$ and $a$ in $\overline{\bar{\sigma}}_{\omega}$ we write $a \star \alpha$ for the concatenation of $a$ and $\alpha$, which is the element $\beta$ of $\sigma_{\omega}$ given by $\bar{\beta}(\lg (a))=a$ and for all $n \in \mathbb{N}: \beta(\lg (a)+n)=\alpha(n)$. For each $a$ in $\overline{\bar{\sigma}}_{\omega}$ we define a canonical element $\alpha_{a}$ of $\sigma_{\omega}$ by putting: $\alpha_{a}=a \star \underline{0}$ where $\underline{0}=0,0,0, \ldots$ We write $\sigma_{\omega} \cap a$ for the set $\left\{\alpha \in \sigma_{\omega} \mid \bar{\alpha}(\lg (a))=a\right\}$ of sequences 'running through $a$ '.

A function $f$ from $\sigma_{\omega}$ to $\mathbb{N}$ is called a spread-function iff there is a function $g$ from $\overline{\bar{\sigma}}_{\omega}$ to $\mathbb{N}$ such that for each $\alpha$ in $\sigma_{\omega}: \exists ! n \in \mathbb{N}[g(\bar{\alpha}(n))>0]$ and moreover for all $n \in \mathbb{N}: g(\bar{\alpha}(n))>0 \rightarrow f(\alpha)=g(\bar{\alpha}(n))-1^{10}$. More generally a function $f$ from $\sigma_{\omega}$ to $\sigma_{\omega}$ is called a spread-function iff there is a function $g$ from $\bar{\sigma}_{\omega}$ to $\bar{\sigma}_{\omega}$ such that for each $\alpha$ in $\sigma_{\omega}$ and $n \in \mathbb{N}$ there is an $m \in \mathbb{N}$ such that: $\overline{f(\alpha)}(n)=g(\bar{\alpha}(m))$, and moreover $g(\bar{\alpha}(n)) \sqsubseteq g(\bar{\alpha}(n+1))$. In both definitions we say that $g$ represents $f$. (Notice that a spread-function is continuous with respect to $d_{\omega}$.).

The fundamental intuitionistic axiom of continuous choice $\mathbf{A C}_{11}$ can now be formulated as follows:

$\mathbf{A C}_{11}$ Let $A$ be a subset of $\sigma_{\omega} \times \sigma_{\omega}$ such that:

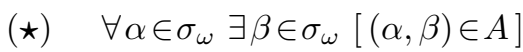

Then there is a spread-function $\gamma$ from $\sigma_{\omega}$ to $\sigma_{\omega}$ such that for each $\alpha$ in $\sigma_{\omega}:(\alpha, \gamma(\alpha))$ is in $A$. We say that $\gamma$ fulfills $(\star)$.

We formulate four weaker versions of this axiom: $\mathbf{A C}_{10}, \mathbf{C P}, \mathbf{A C}_{01}$, and $\mathbf{A C}_{00}$. The last two are simple axioms of countable choice, whereas $\mathbf{A C}_{10}$ is still an axiom of continuous choice, also known as 'Brouwer's principle for numbers'. $\mathbf{A C}_{10}$ implies the so-called continuity principle $\mathbf{C P}$. In section 8 we will indicate two, both classically and intuitionistically valid axioms called $\mathbf{B D D}$ and $\mathbf{A} \mathbf{C}_{11 \text { weak }}$, such that $\mathbf{A} \mathbf{C}_{11}$ is equivalent to the combination of $\mathbf{C P}, \mathbf{B D D}$ and $\mathbf{A} \mathbf{C}_{11 \text { weak }}$. Therefore, $\mathbf{C P}$ may be held solely responsible for intuitionistic results which are not acceptable when interpreted classically ${ }^{1112}$. Also we ask attention for axioms of dependent choice. We do not explain or defend the axioms in this section since they are broadly discussed in the literature (they can all be found in [Kleene\&Vesley1965], [Gielen et al. 1981], [Veldman1981] and [Troelstra\&vanDalen1988]). We begin with the weaker axioms dealing with continuous choice:

\footnotetext{
${ }^{10}$ Notice that $\left\{a \in \overline{\bar{\sigma}}_{\omega} \mid g(a)>0\right\}$ is a decidable thin bar in the sense of the second definition in 8.8. This shows that the concept of spread-function is inherently the same as the concept of a decidable (thin) bar.

${ }^{11}$ We disregard the so-called Brouwer-Kripke axiom, feeling it is not part of mainstream intuitionism.

${ }^{12}$ In a follow-up paper we intend to give a simple classical game-theoretic setting in which all of the intuitionistic axioms hold true. This should provide classical mathematicians with an intuitive understanding of what intuitionism is about.
} 
$\mathbf{A C}_{10}$ Let $A$ be a subset of $\sigma_{\omega} \times \mathbb{N}$ such that:

$(\star \star) \quad \forall \alpha \in \sigma_{\omega} \exists n \in \mathbb{N}[(\alpha, n) \in A]$

Then there is a spread-function $\gamma$ from $\sigma_{\omega}$ to $\mathbb{N}$ such that for each $\alpha$ in $\sigma_{\omega}:(\alpha, \gamma(\alpha))$ is in $A$. We say that $\gamma$ fulfills $(\star \star)$.

CP Let $A$ be a subset of $\sigma_{\omega} \times \mathbb{N}$ such that:

$\forall \alpha \in \sigma_{\omega} \exists n \in \mathbb{N}[(\alpha, n) \in A]$

Then: $\forall \alpha \in \sigma_{\omega} \exists n \in \mathbb{N} \exists m \in \mathbb{N} \forall \beta \in \sigma_{\omega}[\bar{\alpha}(m)=\bar{\beta}(m) \rightarrow(\beta, n) \in A]$.

We present two simple axioms of countable choice in decreasing order of strength:

$\mathbf{A C}_{01}$ Let $A$ be a subset of $\mathbb{N} \times \sigma_{\omega}$ such that:

(*) $\forall n \in \mathbb{N} \exists \alpha \in \sigma_{\omega}[(n, \alpha) \in A]$

Then there is a function $h$ from $\mathbb{N}$ to $\sigma_{\omega}$ such that for each $n \in \mathbb{N}:(n, h(n))$ is in $A$. We say that $h$ fulfills $(*)$.

$\mathbf{A C}_{00}$ Let $A$ be a subset of $\mathbb{N} \times \mathbb{N}$ such that:

$(* *) \quad \forall n \in \mathbb{N} \exists m \in \mathbb{N}[(n, m) \in A]$

Then there is a function $h$ from $\mathbb{N}$ to $\mathbb{N}$ such that for each $n \in \mathbb{N}:(n, h(n))$ is in $A$. We say that $h$ fulfills $(* *)$.

2.2 Finally we present two axioms of dependent choice in decreasing order of strength. For an intuitionistic justification of these axioms we refer the reader to [Waaldijk1996].

DC 1 Let $\delta$ be in $\sigma_{\omega}$, and let $A$ be a subset of $\sigma_{\omega}$. Suppose $R$ is a subset of $A \times A$ such that:

$\delta \in A \wedge \forall \alpha \in A \exists \beta \in A[(\alpha, \beta) \in R]$

Then there is a sequence $\left(\gamma_{n}\right)_{n \in \mathbb{N}}$ of elements of $\sigma_{\omega}$ such that $\gamma_{0}=\delta$ and for each $n \in \mathbb{N}:\left(\gamma_{n}, \gamma_{n+1}\right)$ is in $R$.

$\mathbf{D C}_{0}$ Let $s \in \mathbb{N}$, and let $A$ be a subset of $\mathbb{N}$. Suppose $R$ is a subset of $A \times A$ such that:

$s \in A \wedge \forall n \in A \exists m \in A[(n, m) \in R]$

Then there is an $\alpha$ in $\sigma_{\omega}$ such that $\alpha(0)=s$ and for each $n \in \mathbb{N}:(\alpha(n), \alpha(n+1))$ is in $R$.

2.3 It is now but a small step to formulate the principle of Bar Induction for Decidable bars: $\mathbf{B I}$. A simple definition:

Definition: A subset $A$ of $\overline{\bar{\sigma}}_{\omega}$ is called inductive iff for all $a$ in $\overline{\bar{\sigma}}_{\omega}: \forall n \in \mathbb{N}[a \star n \in A] \rightarrow a \in A$.

$\mathbf{B I}_{\mathbf{D}}$ Let $B$ be a decidable bar on $\sigma_{\omega}$. Suppose $A$ is an inductive subset of $\overline{\bar{\sigma}}_{\omega}$ such that $B \subseteq A$. Then the empty sequence $\varangle \gg$ (of length 0 ) is in $A$. 
REMARK: In classical mathematics $\mathbf{B I}_{\mathbf{D}}$ can be derived from the principle of the excluded middle. The above version of the bar theorem is therefore classically true. In section 8 we derive $\mathbf{B I}_{\mathbf{D}}$ from an axiom called BT ('Brouwer's Thesis') which also holds both in CLASs and INT.

One of the results following from $\mathbf{B I}_{\mathbf{D}}$ is the axiom known as the fan theorem $\mathbf{F T}$. We need a preliminary definition.

DEFinition: Let $\sigma_{2}$ denote the binary fan $\left(\sigma_{2}=\{0,1\}^{\mathbb{N}}\right)$. Write $\overline{\bar{\sigma}}_{2}$ for the set of finite sequences of elements of $\{0,1\}$ (often written like this: $\overline{\bar{\sigma}}_{2}=\{0,1\}^{*}$ ). Then $\overline{\bar{\sigma}}_{2}=\left\{\bar{\alpha}(n) \mid \alpha \in \sigma_{2}, n \in \mathbb{N}\right\}$. A subset $B$ of $\overline{\bar{\sigma}}_{2}$ is called decidable iff $\forall a \in \overline{\bar{\sigma}}_{2}[a \in B \vee a \notin B]$. A subset $B$ of $\overline{\bar{\sigma}}_{2}$ is a bar on $\sigma_{2}$ iff $\forall \alpha \in \sigma_{2} \exists n \in \mathbb{N}[\bar{\alpha}(n) \in B]$.

FT If $B$ is a decidable bar on $\sigma_{2}$, then $B$ contains a finite bar on $\sigma_{2}$ (in other words: then $\left.\exists n \in \mathbb{N} \forall \alpha \in \sigma_{2} \exists m<n[\bar{\alpha}(m) \in B]\right)$.

2.4 The basic axiom in Russ is of course Church's Thesis: 'every sequence of natural numbers is given by a recursive rule' (many results in RUSS already follow from the weaker statement: 'the set of partial functions from $\mathbb{N}$ to $\mathbb{N}$ is countable'). A partial recursive function $\alpha$ from $\mathbb{N}$ to $\mathbb{N}$ will usually be denoted by something like ' $\phi_{e}$ ' where the natural number $e$ is the recursive index of $\alpha$. This recursive index is nothing but the encoding of the finite algorithm which for each $n \in \mathbb{N}$ tries to compute $\alpha(n)$. There is a decidable subset $I(\mathbb{N}, \mathbb{N})$ of $\mathbb{N}$ such that each $e$ in $I(\mathbb{N}, \mathbb{N})$ is a properly formed recursive index of a partial recursive function from $\mathbb{N}$ to $\mathbb{N}$, and vice versa for each partial recursive function $\alpha$

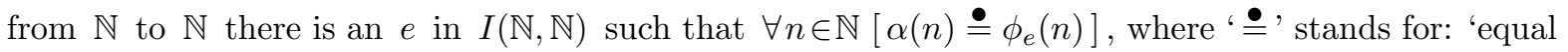
if one of the algorithms terminates, given the input'.

It turns out one can canonically encode each finite recursive computation as a natural number, see [Kleene1952]. This is the basis of Kleene's decidable $T$-predicate on triples of natural numbers $(e, n, k)$, given by:

$T(e, n, k) \Longleftrightarrow e$ is a recursive index and $k$ is the canonical encoding of the computation of $\phi_{e}(n)$.

In particular if $T(e, n, k)$ then the algorithm $\phi_{e}$ terminates on the input $n$. But we are mostly interested in the result of the computation $k$, and in its length (the number of canonical subcomputations leading to the result). Both can be canonically derived from $k$ of course, using recursive funtions Outc and Lgth. So if $T(e, n, k)$, then $\phi_{e}(n)=\operatorname{Outc}(k)$ and the length of the computation $k$ equals $\operatorname{Lgth}(k)$.

In this terminology we formulate Church's thesis thus:

CT $\forall \alpha \in \sigma_{\omega} \exists e \in I(\mathbb{N}, \mathbb{N}) \forall n \in \mathbb{N}\left[\alpha(n)=\phi_{e}(n)\right]$

If $\forall n \in \mathbb{N}\left[\alpha(n)=\phi_{e}(n)\right]$ for $\alpha \in \sigma_{\omega}$ and $e \in I(\mathbb{N}, \mathbb{N})$, then in particular we have: $\forall n \in \mathbb{N} \exists m \in \mathbb{N}[T(e, n, k)]$. Therefore the set $\mathbf{T O T}=\{e \in I(\mathbb{N}, \mathbb{N}) \mid \forall n \in \mathbb{N} \exists k \in \mathbb{N}[T(e, n, k)]\}$ plays an important role in Russ.

The combination of $\mathbf{C T}$ with $\mathbf{A C}_{00}$ is equivalent to an axiom known as $\mathbf{C} \mathbf{T}_{0}$, which illustrates the connection between CT and choice axioms (cf. 8.13):

$\mathbf{C T}_{0}$ Let $A$ be a subset of $\mathbb{N} \times \mathbb{N}$ such that:

$\forall n \in \mathbb{N} \exists m \in \mathbb{N}[(n, m) \in A]$ 
Then there is a recursive function $h$ from $\mathbb{N}$ to $\mathbb{N}$ such that for each $n \in \mathbb{N}:(n, h(n))$ is in $A$.

From $\mathbf{C T}_{0}$ we can derive a more complex choice axiom $\mathbf{C T}_{01}$, which plays a part in our discussion later on:

$\mathbf{C T}_{01}$ Let $A$ be any subset of $\mathbb{N} \times \mathbb{N}$, and $B$ a decidable subset of $\mathbb{N} \times \mathbb{N}$ such that:

$\forall n \in \mathbb{N}[\exists y \in \mathbb{N}[(n, y) \in B] \rightarrow \exists m \in \mathbb{N}[(n, m) \in A]]$

Then there is a partial recursive function $h$ from $\mathbb{N}$ to $\mathbb{N}$ such that for each $n \in \mathbb{N}:$ if $\exists y \in \mathbb{N}[(n, y) \in B]$ then $h(n)$ is defined and $(n, h(n))$ is in $A$.

LEMMA: $\mathbf{C T}_{01}$ follows from $\mathbf{C \mathbf { T } _ { 0 }}$

PROOF: We have by the assumption in $\mathbf{C T}_{01}$ :

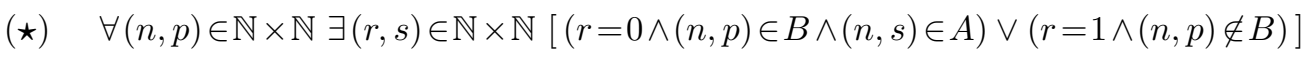

Now identify $\mathbb{N} \times \mathbb{N}$ with $\mathbb{N}$ and apply $\mathbf{C T}_{0}$ to find a recursive function $f$ from $\mathbb{N} \times \mathbb{N}$ to $\mathbb{N} \times \mathbb{N}$ fulfilling $(\star)$. The desired partial recursive $h$ can be defined by describing its working on $n$ : let $h(n)$ be the second coordinate of $f(n, p)$ where $p$ is the least $m \in \mathbb{N}$ for which the first coordinate of $f(n, m)$ equals 0

$\mathbf{C T}_{01}$ is the first step to an even broader choice axiom known as 'Extended Church's Thesis' $\left(\mathbf{E C T}_{0}\right)$, which is widely accepted in RUSS. But the formulation of $\mathbf{E} \mathbf{C} \mathbf{T}_{0}$ and its defense are in logical terms and do not appeal to the author. We will present a simpler version with an intuitive defense in 8.13, but first we present $\mathbf{E C T}_{0}$. A subset $A$ of $\mathbb{N}$ is called almost negative iff for each $n \in \mathbb{N}$ the membership of $A$ is equivalent to the truth of a logical formula $\mathrm{A}(n)$, where A contains the existential quantifier $\exists$ only directly in front of prime formulas, and furthermore is built only from the logic connectives $\rightarrow$ and $\wedge$ and the universal quantifier $\forall$. Notice that in particular the set TOT is almost negative.

$\mathbf{E C T}_{0}$ Let $A$ be any subset of $\mathbb{N} \times \mathbb{N}$, and $B$ an almost negative subset of $\mathbb{N}$ such that:

$\forall n \in \mathbb{N}[n \in B \rightarrow \exists m \in \mathbb{N}[(n, m) \in A]]$

Then there is a partial recursive function $h$ from $\mathbb{N}$ to $\mathbb{N}$ such that for each $n \in B$ we have: $h(n)$ is defined and $(n, h(n))$ is in $A$.

2.5 The second important axiom in RUSS is called Markov's Principle: 'if it is impossible that a total recursive function $\alpha$ does not achieve the value 1 for some $n$ in $\mathbb{N}$, then there is an $n$ in $\mathbb{N}$ with $\alpha(n)=1$ '. Formally:

MP Let $\alpha$ be in $\sigma_{\omega}$ such that $\neg \neg \exists n \in \mathbb{N}[\alpha(n)=1]$. Then $\exists n \in \mathbb{N}[\alpha(n)=1]$.

The motivation in RUSS is as follows. Existence of an object ('there is') in constructive mathematics always denotes a method to come by this object. Having an algorithm for computing $\alpha(n)$ for each $n \in \mathbb{N}$, and knowing it cannot for all $n \in \mathbb{N}$ avoid returning the value 1 , we can simply wait until this algorithm comes up with the desired $n$. In other words the method here is 'wait'. This reasoning seems valid outside the recursive context also, nevertheless Markov's Principle is not generally accepted 
in INT. The usual reason given for not accepting MP is that MP does not enable one to fix a prior bound on the number of computations needed. However, this reason can be questioned. Suppose that to deserve the predicate 'there is' one must give a prior bound to the number of computations necessary. For a recursive $\alpha$, the complexity of giving a prior bound to the number of computations needed for computing $\alpha(n)$ is not less than the complexity of $\alpha$ itself. So to show 'there is' a prior bound, one must give a prior bound for the number of computations needed to give a prior bound, and this task is at least as hard as simply computing $\alpha$. And then one must give a prior bound for giving the prior bound for giving the prior bound etc. Therefore giving a prior bound is not less complex than letting $\alpha$ run by itself.

Also the complexity of natural induction (see Ind in 2.6) is such that in general one cannot indicate beforehand a bound on the number of computations needed to complete the induction. One can only show that the complexity is reduced by one step, with each step. So really one in general has no other method to evaluate an inductive definition or computation than to wait. But when thinking it through, this is precisely the same as what happens in the waiting process when we let $\alpha$ run. So the intuition behind Ind is not different from the intuition behind MP.

This extends to the elements of the non-recursive intuitionistic universe just as well. Even though an element $\alpha$ of $\sigma_{\omega}$ need not be recursive, the reasoning above still applies. So in the author's eyes it is worthwile to consider adopting MP in intuitionism as well.

DEFINITION: Since recursion theory also is a part of CLASS and INT, we can prove most theorems concerning RUSS in a constructive recursion theoretic setting. We therefore use terminology such as $\sigma_{\omega \mathrm{REC}}, \sigma_{2 \mathrm{REC}}$, etcetera to indicate the subset of recursive sequences in $\sigma_{\omega}, \sigma_{2}$, etcetera.

2.6 Finally we present the principle of induction as an axiom. This completes our presentation of basic axioms. More axioms will be discussed in section 8 .

Ind Let $A$ be a subset of $\mathbb{N}$ such that $0 \in A$ and for all $n \in \mathbb{N}: n \in A$ implies $n+1 \in A$. Then $A=\mathbb{N}$, that is: $n \in A$ for all $n \in \mathbb{N}$.

\section{BASIC DEFINITIONS REGARDING CONTINUOUS FUNCTIONS}

3.1 We assume the reader to be familiar with the (constructive and/or intuitionistic and/or even classical) definition of the real numbers, 'compact metric space', etcetera. Only those definitions which can be considered special or specifically relevant will be repeated here. We turn immediately to the definition of '(uniformly) continuous function'. We believe the following to be the most fruitful definition in the context of metric spaces.

DEFINITION: Let $f$ be a function from a metric space $(X, d)$ to another metric space $\left(Y, d_{Y}\right)$. Then $f$ is continuous iff for all $x \in X$ and all $n \in \mathbb{N}$ there is an $m \in \mathbb{N}$ such that for all $z \in B\left(x, 2^{-m}\right)$ we have: $f(z) \in B\left(f(x), 2^{-n}\right)$. We say that $f$ is uniformly continuous iff for all $n \in \mathbb{N}$ there is an $m \in \mathbb{N}$ such that for all $x \in X$ and all $z \in B\left(x, 2^{-m}\right)$ we have: $f(z) \in B\left(f(x), 2^{-n}\right)$. 
Furthermore we will be sloppy with our notation of the well-known metric spaces involved, for instance writing $\mathbb{R}^{+}$instead of $\left(\mathbb{R}^{+}, d_{\mathbb{R}}\right)$ whenever confusion is unlikely. We do not advocate this in general, but within the simple scope of this paper mistakes will not occur.

3.2 Now let us give the conditions (2)(a)-(d) in theorem 1.3 a more precise name and place.

DEFINITION: We define a number of 'continuity properties' which a given class of continuous functions $\mathcal{K}$ may or may not possess:

Cont I If $f$ is a uniformly continuous function, then $f$ is in $\mathcal{K}$

Cont II If $f$ and $g$ are functions in $\mathcal{K}$ such that $\operatorname{Ran}(f) \subseteq \operatorname{Dom}(g)$, then the composition $g \circ f$ is in $\mathcal{K}$

Cont III If $f$ is a function in $\mathcal{K}$ defined on a compact space, then $f$ is uniformly continuous

Cont IV The function $x \longmapsto \frac{1}{x}$, defined on $\mathbb{R}^{+}$, is in $\mathcal{K}$

We will study these properties in section 5 . To strengthen our results we will also study slightly weaker variants of these properties, such as restricting Cont I to uniformly continuous functions defined on $[0,1]$. In theorem 1.3 we also restrict Cont III to functions defined on $[0,1]$. Disregarding such subtle strengthenings theorem 1.3 can be rephrased thus: the existence of a class of functions satisfying Cont I through Cont IV implies FT, and vice versa.

3.3 Bishop's 1967 definition of 'continuous function' will be called 'continuous ${ }^{\mathrm{BIs}}$ function' in this paper. The definition is limited to functions $f$ such that $\operatorname{Dom}(f)$ and $\operatorname{Ran}(f)$ are subspaces of the real numbers $\mathbb{R}$, and is given directly below. The class of continuous ${ }^{\mathrm{BIS}}$ functions is easily seen to satisfy Cont I, Cont III and Cont IV.

DEFINITION: Let $f$ be a real-valued function defined on a subspace $X$ of $\mathbb{R}$. Then $f$ is continuous ${ }^{B I S}$ iff $f$ is continuous, and uniformly continuous on each compact subspace of $X$.

Already at its birth it was seen that this definition has a possibly serious drawback: how to prove within BISH that, when defined, the composition of two arbitrary continuous ${ }^{\mathrm{BIS}}$ functions is again continuous ${ }^{\mathrm{BIS}}$ ? In other words, does the class of continuous ${ }^{\mathrm{BIS}}$ functions satisfy Cont II? Bishop proposed a lemma of convenience, rather than answering the question with theorem 1.3. It follows from this theorem however that the lemma doesn't resolve the fundamental problem. We repeat this lemma for completeness' sake, leaving the simple proof to the reader.

LEMMA: (Bishop 1967) Let $f$ be a real-valued continuous ${ }^{\mathrm{BIS}}$ function defined on a subspace $X$ of $\mathbb{R}$. Let $g$ be a real-valued continuous ${ }^{\text {BIs }}$ function defined on a subspace $Y$ of $\mathbb{R}$ such that $\operatorname{Ran}(f) \subseteq Y$. Suppose $f$ maps each compact subspace of $X$ into a compact subspace of $Y$. Then the composition $g \circ f$ is again continuous ${ }^{\mathrm{BIS}}$.

Still a second drawback was discovered later: how to extend the [Bishop1967] definition 'continuous ${ }^{\mathrm{BIS}}$, to metric spaces in general, not just $\left(\mathbb{R}, d_{\mathbb{R}}\right)$ ? For locally compact spaces there is no problem, simply 
drop the conditions 'real-valued' and 'subspace of $\mathbb{R}$ '. But if the resulting definition (which we also call continuity $^{\mathrm{BIS}}$ for simplicity) is also used for non-locally-compact spaces, one loses a desirable property in the process. For given a continuous ${ }^{\mathrm{BIS}}$ function $f$ from a metric space $(X, d)$ to another metric space $\left(Y, d_{Y}\right)$, and given a compact subspace $(Z, d)$ of $(X, d)$, we do not see how to conclude in general from the given definition that:

(*) $\forall n \in \mathbb{N} \exists m \in \mathbb{N} \forall z \in Z \forall x \in X\left[d(x, z)<2^{-m} \rightarrow d_{Y}(f(x), f(z))<2^{-n}\right]$

If one wishes for continuous functions which are uniformly continuous on compact subspaces, then $(\star)$ seems even better, and is true both in classical and intuitionistic mathematics. Actually the pragmatic solution would be to simply add $(\star)$ to the definition.

3.4 An attempt to repair the two drawbacks mentioned above is found in [Bridges1979], where an alternative definition of 'continuous function' (which we call 'continuous ${ }^{\text {BRI }}$ function' ) is given. When defined, the composition of two continuous ${ }^{\mathrm{BRI}}$ functions is always a continuous ${ }^{\mathrm{BRI}}$ function. Every continuous $^{\mathrm{BRI}}$ function is continuous ${ }^{\mathrm{BIS}}$. To understand the [Bridges1979] definition we need a preliminary.

DEFINITION: Let $(X, d)$ be a metric space. Then $(X, d)$ is called a compact image iff $(X, d)$ is the uniformly-continuous image of the binary fan $\left(\sigma_{2}, d_{\omega}\right)$ (for $d_{\omega}$ see def. 2.1).

REMARK: This is easily seen to be equivalent to the more complicated definition in [Bridges1979], if we keep in mind that every compact space is the uniformly-continuous image of the binary fan $\left(\sigma_{2}, d_{\omega}\right)^{13}$. The finer constructive view finds a distinction between uniformly-continuous images of $\left(\sigma_{2}, d_{\omega}\right)$ which are metrically complete (and then called compact) and uniformly-continuous images of $\left(\sigma_{2}, d_{\omega}\right)$ which are not necessarily complete. Classically all such spaces are compact themselves, and the above definition is superfluous. Intuitionistically one can prove many a compact image to be metrically incomplete, yet it is possible to develop an attractive general topological theory, with a non-metrical (but metrizable) concept of 'compact' such that the continuous image of a compact space is again compact. This theory closely parallels the classical approach, and depends on FT (see [Waaldijk1996]).

3.5 The [Bridges1979] definition now runs like this:

DEFINITION: Let $f$ be a function from a metric space $(X, d)$ to another metric space $\left(Y, d_{Y}\right)$. Let $(Z, d)$ be a subspace of $(X, d)$. We say that $f$ is uniformly continuous near $(Z, d)$ iff

$\forall n \in \mathbb{N} \exists m \in \mathbb{N} \forall z \in Z \forall x \in X\left[d(x, z)<2^{-m} \rightarrow d_{Y}(f(x), f(z))<2^{-n}\right]$

We say that $f$ is continuous ${ }^{B R I}$ iff $f$ is uniformly continuous near each compact image contained in $(X, d)$.

REMARK: Notice that trivially every continuous ${ }^{\text {BRI }}$ function is continuous ${ }^{\text {BIS }}$. So far no general choice has been made between these two definitions. In [Bishop\&Bridges1985] the [Bishop1967] definition is repeated, likewise in [Bridges\&Richman1987]. It would seem that the [Bishop1967] definition is favoured, but there is no written motivation for this that the author knows of. Meanwhile, the two definitions

\footnotetext{
${ }^{13}$ This topological fact was already proven by Brouwer (see the collected works [Brouwer1975]).
} 
are not equivalent, and a theorem proved using one definition should be checked to see whether it still holds under the other definition.

One easily sees that the class of continuous ${ }^{\mathrm{BRI}}$ functions satisfies Cont I through Cont III. Therefore, as corollary 1.3 points out, the assertion of Cont IV for the class of continuous ${ }^{\mathrm{BRI}}$ functions is equivalent to $\mathbf{F T}^{14}$.

\section{ProOF OF THEOREM 1.3}

4.1 The following lemma will play a nice role in this paper.

LEMma: Let $f$ be a uniformly continuous function from $\left(\sigma_{2}, d_{\omega}\right)$ to $\left(\mathbb{R}, d_{\mathbb{R}}\right)$, then there is a decidable subset $B$ of $\overline{\bar{\sigma}}_{2}$ such that:

(i) $\forall \alpha \in \sigma_{2} \forall n \in \mathbb{N}\left[\bar{\alpha}(n) \in B \rightarrow f(\alpha)>2^{-n}\right]$

(ii) $\forall \alpha \in \sigma_{2}[f(\alpha)>0 \rightarrow \exists m \in \mathbb{N}[\bar{\alpha}(m) \in B]]$.

PROOF: Since $f$ is uniformly continuous we have:

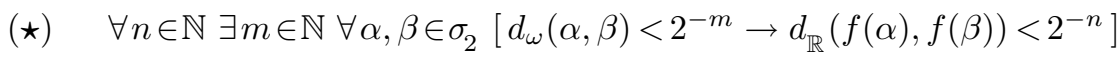

By $\mathbf{A C}_{00}$ there is a function $u$ from $\mathbb{N}$ to $\mathbb{N}$ fulfilling $(\star)$. Without loss of generality this function is such that for all $n \in \mathbb{N}: u(n+1)>u(n) \geq n$. For each $n \in \mathbb{N}$ and each $\alpha$ in $\sigma_{2}$ at least one of the two following statements is true: $f(\alpha)>2^{-n+1}$ or $f(\alpha)<2^{-n}$. Therefore we find (with $\alpha_{a}=a \star \underline{0}$ see definition 2.1):

$(\star \star) \quad \forall n \in \mathbb{N} \forall a \in \bar{\sigma}_{2} \exists t \in\{0,1\}\left[\left(t=0 \wedge f\left(\alpha_{a}\right)>2^{-n}\right) \vee\left(t=1 \wedge f\left(\alpha_{a}\right)<2^{-n+1}\right)\right]$

By $\mathbf{A C}_{00}$ there is a function $v$ from $\mathbb{N} \times \bar{\sigma}_{2}$ to $\{0,1\}$ fulfilling ( $\left.\star \star\right)$. We are ready to define the desired decidable $B$ which satisfies the lemma, as follows. Put:

$B=\left\{a \in \sigma_{2} \mid \exists s<\lg (a)[v(s, a)=0 \wedge u(s+1)<\lg (a)]\right\}$

Clearly $B$ is decidable, it remains to show that $\forall \alpha \in \sigma_{2} \forall n \in \mathbb{N}\left[\bar{\alpha}(n) \in B \rightarrow f(\alpha)>2^{-n-1}\right]$ and $\forall \alpha \in \sigma_{2}[f(\alpha)>0 \rightarrow \exists m \in \mathbb{N}[\bar{\alpha}(m) \in B]]$. So first let $\alpha$ in $\sigma_{2}$ and $n \in \mathbb{N}$ and suppose $a=\bar{\alpha}(n) \in B$. Then there is $s<\lg (a)=n$ such that $v(s, a)=0$. Therefore $f\left(\alpha_{a}\right)>2^{-s}$ and also $u(s+1)<\lg (a)=n$, therefore $d_{\omega}\left(\alpha, \alpha_{a}\right)<2^{-u(s+1)}$ and so $d_{\mathbb{R}}\left(f(\alpha), f\left(\alpha_{a}\right)\right)<2^{-s-1}$ and so $f(\alpha)>2^{-s-1} \geq 2^{-n}$. Now finally let $\alpha$ in $\sigma_{2}$ be such that $f(\alpha)>0$. Determine $s \in \mathbb{N}$ such that $f(\alpha)>2^{-s+2}$. Determine $t=u(s+1)$ and put $a=\bar{\alpha}(t+1)$, then $d_{\omega}\left(\alpha, \alpha_{a}\right)<2^{-u(s+1)}$ and so $f\left(\alpha_{a}\right)>2^{-s+1}$. Therefore $v(s, a)=0$. Since $u(s+1)=t<\lg (a)$ we see that $a$ is in $B$

As a direct consequence we obtain the following proposition, already proven in [Beeson1985], which plays a central part in our proof of theorem 1.3:

PROPOSITION: Within BISH the following statements are equivalent:

\footnotetext{
${ }^{14}$ This is due to the clause 'each compact image' in the definition of continuity ${ }^{\mathrm{BRI}}$. Replace this with 'each compact subspace', and one gets an improvement of continuity ${ }^{\mathrm{BIS}}$, losing Cont II in the process.
} 
(1) The fan theorem $\mathbf{F T}$.

(2) If $f$ is a uniformly continuous function from $\left(\sigma_{2}, d_{\omega}\right)$ to $\left(\mathbb{R}^{+}, d_{\mathbb{R}}\right)$, then there is an $N \in \mathbb{N}$ such that for all $\alpha$ in $\sigma_{2}: f(\alpha)>2^{-N}$.

PROOF: To prove $(1) \Rightarrow(2)$ assume $\mathbf{F T}$ and let $f$ be a uniformly continuous function from $\sigma_{2}$ to $\mathbb{R}^{+}$. By the above lemma there is a decidable bar (!) $B$ on $\sigma_{2}$ such that $\forall \alpha \in \sigma_{2} \forall n \in \mathbb{N}[\bar{\alpha}(n) \in B \rightarrow$ $\left.f(\alpha)>2^{-n-1}\right]$. By FT we have that $B$ contains a finite bar on $\sigma_{2}$, in other words there is an $N \in \mathbb{N}$ such that $\forall \alpha \in \sigma_{2} \exists m<N[\bar{\alpha}(m) \in B]$. Therefore for all $\alpha$ in $\sigma_{2}$ we have: $f(\alpha)>2^{-N}$.

To prove $(2) \Rightarrow(1)$ assume (2) and let $B$ be a decidable bar on $\sigma_{2}$. Define a uniformly continuous function $f$ from $\sigma_{2}$ to $\mathbb{R}^{+}$by putting, for $\alpha$ in $\sigma_{2}: f(\alpha)=2^{-s}$ where $s=\mu n \in \mathbb{N}[\bar{\alpha}(n) \in B]$ (' $\mu n \in \mathbb{N}$ ' means: 'the smallest $n \in \mathbb{N}$ such that'). Then by (2) there is an $N \in \mathbb{N}$ such that all $\alpha$ in $\sigma_{2}$ : $f(\alpha)>2^{-N}$. Clearly this implies that $\forall \alpha \in \sigma_{2} \exists m<N[\bar{\alpha}(m) \in B]$, in other words $B$ contains a finite bar $\bullet$

4.2 The second observation that we will use is the one-to-one correspondence of $\sigma_{2}$ and the Cantor space $\mathcal{C}$, which is the well-known compact subspace of $[0,1]$ given by the formula:

$\mathcal{C}=\left\{\sum_{n \in \mathbb{N}} 2 \alpha(n) \cdot 3^{-n-1} \mid \alpha \in \sigma_{2}\right\}$

The one-to-one correspondence is of course given by the uniformly continuous function $j_{\mathcal{C}}$ given by $j_{\mathcal{C}}(\alpha)=\sum_{n \in \mathbb{N}} 2 \alpha(n) \cdot 3^{-n-1}$ for $\alpha$ in $\sigma_{2}$, with the uniformly continuous inverse $j_{\mathcal{C}}^{-1}$.

PROPOSITION: Within BISH the following statements are equivalent:

(1) The fan theorem $\mathbf{F T}$.

(2) If $f$ is a uniformly continuous function from $[0,1]$ to $\mathbb{R}^{+}$, then there is an $N \in \mathbb{N}$ such that for all $x$ in $[0,1]: f(x)>2^{-N}$.

PROOF: The implication $(1) \Rightarrow(2)$ is shown as follows. Assume $\mathbf{F T}$, and let $f$ be a uniformly continuous function from $[0,1]$ to $\mathbb{R}^{+}$. Let $g$ be a uniformly continuous surjection from $\sigma_{2}$ to $[0,1]$. Then $h=f \circ g$ is a uniformly continuous function from $\sigma_{2}$ to $\mathbb{R}^{+}$, so by proposition 4.1 there is an $N \in \mathbb{N}$ such that for all $\alpha$ in $\sigma_{2}: h(\alpha)>2^{-N}$. We conclude that for all $x$ in $[0,1]: f(x)>2^{-N}$. To prove $(2) \Rightarrow(1)$ assume (2) and let $B$ be a decidable bar on $\sigma_{2}$. Define a uniformly continuous function $h$ from $\sigma_{2}$ to $\mathbb{R}^{+}$by putting, for $\alpha$ in $\sigma_{2}: h(\alpha)=2^{-s}$ where $s=\mu n \in \mathbb{N}[\bar{\alpha}(n) \in B]$. Clearly by the above one-to-one correspondence between $\sigma_{2}$ and $\mathcal{C}$ we can define a corresponding uniformly continuous function $g$ from $\mathcal{C}$ to $\mathbb{R}^{+}$by putting $g=h \circ j_{\mathcal{C}}^{-1}$. By the Tietze extension theorem (see [Bishop1967]) or simply by linear interpolation $g$ can be extended to a uniformly continuous function $f$ from $[0,1]$ to $\mathbb{R}^{+}$. Then by (2) there is an $N \in \mathbb{N}$ such that for all $x$ in $[0,1]: f(x)>2^{-N}$. So for all $x$ in $\mathcal{C}$ we have: $g(x)>2^{-N}$. Clearly this implies that for all $\alpha$ in $\sigma_{2}: h(\alpha)>2^{-N}$, which in turn implies that $\forall \alpha \in \sigma_{2} \exists m<N[\bar{\alpha}(m) \in B]$, in other words $B$ contains a finite bar

4.3 We are ready to prove the main theorem 1.3, which we repeat here for the reader's convenience:

Theorem: (repeated from 1.3) Within BISH the following statements are equivalent:

(1) The fan theorem FT. 
(2) There exists a class of real-valued functions called 'kontinuous' functions such that:

(a) If $f$ is a uniformly continuous real-valued function defined on $[0,1]$, then $f$ is kontinuous.

(b) If $f$ and $g$ are kontinuous functions such that $\operatorname{Ran}(f) \subseteq \operatorname{Dom}(g)$, then the composition $g \circ f$ is kontinuous.

(c) If $f$ is a kontinuous function defined on $[0,1]$, then $f$ is uniformly continuous.

(d) The function $x \longmapsto \frac{1}{x}$, defined on $\mathbb{R}^{+}$, is kontinuous.

PROOF: The implication (1) $\Rightarrow(2)$ follows by taking for 'kontinuous' the class of continuous ${ }^{\mathrm{BRI}}$ functions. Assume FT, then for this class all but (2)(d) follow immediately. To prove (2)(d) we use proposition 4.1, which says that $\mathbf{F T}$ implies that every compact image in $\mathbb{R}^{+}$has a positive distance from 0 . Therefore the function $x \longmapsto \frac{1}{x}$ is uniformly continuous near every compact image, and thus a continuous ${ }^{\mathrm{BRI}}$ function.

Now for the implication $(2) \Rightarrow(1)$. Suppose we have a class of real-valued functions called 'kontinuous' functions such that (2)(a)-(d) hold. We need to derive from this the fan theorem FT. By proposition 4.2 it suffices to show:

( $\star$ If $f$ is a uniformly continuous function from $[0,1]$ to $\mathbb{R}^{+}$, then there is an $N \in \mathbb{N}$ such that for all $x$ in $[0,1]: f(x)>2^{-N}$.

Therefore let $f$ be a uniformly continuous function from $[0,1]$ to $\mathbb{R}^{+}$. By $(2)(a) f$ is kontinuous. By (2)(d) and (2)(b) the composition function $h$ defined by $x \stackrel{h}{\longmapsto} \frac{1}{f(x)}$ is kontinuous. Therefore by $(2)(c) h$ is a uniformly continuous real-valued function defined on $[0,1]$. This in turn means that there is an $N \in \mathbb{N}$ such that for all $x$ in $[0,1]: h(x)=\frac{1}{f(x)}<2^{N}$. But then of course for all $x$ in $[0,1]$ : $f(x)>2^{-N}$. Thus we have shown $(\star)$, and by proposition 4.2 this implies FT $\bullet$

4.4 Since at least corollary 1.3 should elicit some surprise, we will prove it explicitly. This will also clarify the applicability of the main theorem itself. For the reader's sake we repeat the corollary here:

COROLLARY: (repeated from 1.3) Within BISH the following three statements are equivalent:

(1) The fan theorem FT.

(2) If $f$ is a continuous ${ }^{\mathrm{BIS}}$ function from $[0,1]$ to $\mathbb{R}^{+}$, then the composition $x \longmapsto \frac{1}{f(x)}$ of $f$ with the continuous ${ }^{\mathrm{BIS}}$ function $x \longmapsto \frac{1}{x}$ is continuous ${ }^{\mathrm{BIS}}$.

(3) The function $x \longmapsto \frac{1}{x}$, defined on $\left(\mathbb{R}^{+}, d_{\mathbb{R}}\right)$, is continuous ${ }^{\mathrm{BRI}}$.

PROOF: The implication (3) $\Rightarrow(1)$ is shown as follows. Suppose (3) holds, then the class of continuous ${ }^{\mathrm{BRI}}$ functions satisfies (2)(a)-(d) of theorem 1.3. The easy verification of this is left to the reader. So taking 'kontinuous' to mean 'continuous ${ }^{\mathrm{BRI}}$ ' we obtain (2) of theorem 1.3, which by the theorem implies FT. For the implication $(1) \Rightarrow(3)$ we use proposition 4.1 , which says that FT implies that every compact image in $\mathbb{R}^{+}$has a positive distance from 0 . Therefore the function $x \longmapsto \frac{1}{x}$ is uniformly continuous near every compact image, and thus a continuous ${ }^{\mathrm{BRI}}$ function.

Now for the implication $(2) \Rightarrow(1)$. Suppose (2) holds, then we must deduce FT. However, using (2) we can pinpoint a subclass $\mathcal{K}$ of the class of continuous $^{\mathrm{BIS}}$ functions, such that this subclass satisfies 
(2)(a)-(d) of theorem 1.3. To this end let $\mathcal{K}$ be the class of functions which contains precisely all uniformly continuous real-valued functions with domain $[0,1]$ and also the functions $x \longmapsto \frac{1}{x}$ and the identity $x \longmapsto x$ both with domain $\mathbb{R}^{+}$. Trivially the class $\mathcal{K}$ satisfies (2)(a), (2)(c) and (2)(d). To show that (2)(b) holds as well, let $f$ and $g$ be in $\mathcal{K}$ such that $\operatorname{Ran}(f) \subseteq \operatorname{Dom}(g)$. It remains to show that the composition $g \circ f$ is also in $C$. If both $f$ and $g$ are equal to one of the functions $x \longmapsto \frac{1}{x}$ or $x \longmapsto x$ (with domain $\mathbb{R}^{+}$) then we are done, the composition is again one of these functions. If both $f$ and $g$ have as domain $[0,1]$, then by definition of $\mathcal{K}$ they are both uniformly continuous, therefore their composition $g \circ f$ is again a uniformly continuous function with domain $[0,1]$, and so in $C$. The only other possible case is that $f$ is a uniformly continuous function with domain $[0,1]$, and $g$ is either $x \longmapsto \frac{1}{x}$ or the identity $x \longmapsto x$ (with domain $\mathbb{R}^{+}$). If $g$ is the identity then we are done, the composition trivially is in $C$. If finally $g$ is $x \longmapsto \frac{1}{x}$, then the statement that the composition $x \longmapsto \frac{1}{f(x)}$ of $f$ with the function $x \longmapsto \frac{1}{x}$ is continuous ${ }^{\mathrm{BIS}}$ and therefore uniformly continuous is precisely the assumption (2). So the class $\mathcal{K}$ satisfies (2)(a)-(d) of theorem 1.3, and by this theorem this implies the fan theorem FT.

The implication $(1) \Rightarrow(2)$ is shown as follows. Assume $\mathbf{F T}$, and let $f$ be a uniformly continuous function from $[0,1]$ to $\mathbb{R}^{+}$. By proposition 4.2 there is an $N \in \mathbb{N}$ such that for all $x$ in $[0,1]: f(x)>2^{-N}$. But of course the function $x \longmapsto \frac{1}{x}$ is uniformly continuous on $\left\{x \in \mathbb{R}^{+} \mid x>2^{-N}\right\}$, therefore the composition $x \longmapsto \frac{1}{f(x)}$ of $f$ with the function $x \longmapsto \frac{1}{x}$ is uniformly continuous and therefore continuous ${ }^{\mathrm{BIS}} \bullet$

\section{Possible alternative Definitions?}

5.1 In the author's opinion, theorem 1.3 and its corollary show that in the absence of the axiom FT the possibilities for an elegant definition of 'continuous function' which allows one to constructively reproduce a general part of classical mathematics are limited. To clarify this opinion, we briefly investigate the properties that a class of 'continuous functions' can be stated to have without (by this stating) implying the fan theorem. Theorem 1.3 says this is only possible by (partly) dropping or amending the properties Cont I through Cont IV for the then-defined class of continuous functions. Let's review some alternatives one at a time.

The first and very obvious alternative is to drop Cont III altogether, and possibly allow for continuous functions on $[0,1]$ which are not uniformly continuous. This amounts to the normal definition of 'continuous function' (which we also adopt, see 3.1). However one of Bishop's ends was to constructively reproduce a certain general part of classical mathematics, namely continuous functions being uniformly continuous on compact (sub)spaces. So in our opinion amending Cont III means changing the spirit of BISH. Still, this could be quite acceptable. And the connection with classical, intuitionistic and recursive mathematics becomes simplified, since the definitions can be the same.

REMARK: Notice the current role of BISH with respect to recursive mathematics. There are many interesting continuous recursive functions on the recursive continuum $\left([0,1]_{\mathrm{REC}}\right)$ which are not uniformly continuous and therefore not continuous ${ }^{\mathrm{BIS}}$ or continuous ${ }^{\mathrm{BRI}}$. This in turn means that these functions are currently not studied as continuous functions in BISH, whereas BISH claims to be a neutral playground for recursive, intuitionistic and classical mathematics. This leads to the question whether the current 
focus of BISH is any different than the focus determined by FT.

Another alternative is to amend or drop Cont IV. This alternative corresponds in fact to the solution obtained with the definition of continuity ${ }^{\mathrm{BRI}}$, see corollary 1.3. The author of this article does not believe this to be a viable alternative. More to the point: the author doesn't believe that a perfectly beautiful constructive function such as $x \longmapsto \frac{1}{x}$ can seriously be doubted to be continuous. Such a doubt, if fundamental, reveals a lack of elegance in definitions or axioms which must avenge itself in all sorts of unforeseen ways, when more of mathematics will be constructively charted. The function $x \longmapsto \frac{1}{x}$ for example plays a crucial part in complex analysis and the theory of Riemannian manifolds, where topology is all-pervasive.

Next we consider partly dropping or amending Cont II. This alternative corresponds to the solution obtained with the definition of continuity ${ }^{\mathrm{BIS}}$, see corollary 1.3 (however the definition of continuity ${ }^{\mathrm{BIS}}$ should be improved on, see 3.3). If one wishes to retain the spirit of BISH, then this alternative seems the least problematic. In the field of analysis the author cannot straightaway pinpoint troublesome consequences arising from the uncertainty whether the composition of two continuous functions is again continuous. ${ }^{15}$ He believes this to be partly because not enough of classical mathematics has been charted constructively. Once this is done, the author believes that the uncertainty about Cont II will be seen to create many problems for elegance and generality, especially in the field of topology. The pragmatist however need not necessarily care.

Finally there is the possibility of dropping or amending condition Cont I. One must then find a subclass 'kontinuous' of the class of continuous functions for which Cont II through Cont IV hold. Then Cont I for this class becomes equivalent to the fan theorem. So then without FT one cannot prove that all uniformly continuous functions defined on $[0,1]$ are 'kontinuous'. We will investigate this possibility in some detail in order to explain why the author thinks it doesn't work. Some interesting results are obtained in the process.

5.2 Roughly speaking, amending Cont I comes down to separating the uniformly continuous functions on $\left(\sigma_{2}, d_{\omega}\right)$ (or equivalently on $\mathcal{C}$, or even on $\left.[0,1]\right)$ in two classes: the 'kontinuous' class and the 'notkontinuous' class. However, in order to do nice mathematics the kontinuous class should contain enough functions. Therefore 'nice' uniformly continuous functions should certainly be kontinuous. Let us for a moment extend our view to include $\mathbb{R}^{2}$ and $\mathbb{R}^{2}$-valued functions as well. Then we feel that given any element $z$ of $\mathbb{R}^{2}$ the distance function $d_{z}$ given by $d_{z}(w)=d_{\mathbb{R}^{2}}(z, w)$ (for all $w$ in $\mathbb{R}^{2}$, where $d_{\mathbb{R}^{2}}$ is the Euclidean distance function) should be kontinuous. Given this and Cont II through Cont IV we will show that there is a single uniformly continuous function $f_{\mathrm{bar}}$ from $\sigma_{2}$ to $\mathbb{R}^{2}$ such that the kontinuity of $f_{\text {bar }}$ implies the fan theorem.

5.3 The definition of this $f_{\text {bar }}$ hinges on the possibility to encode an arbitrary decidable subset $B$ of $\overline{\bar{\sigma}}_{2}$ with an element $\alpha_{B}$ of $\sigma_{2}$, as follows. Fix (for this section) a bijection $h$ from $\mathbb{N}$ to $\overline{\bar{\sigma}}_{2}$, with inverse $h^{-1}$. Let $B$ be a decidable subset of $\overline{\bar{\sigma}}_{2}$, then $\alpha_{B}$ is given by:

$\alpha_{B}(n) \overline{\bar{D}}\left\{\begin{array}{ll}0 & \text { if } h(n) \notin B \\ 1 & \text { if } h(n) \in B\end{array} \quad(\right.$ for all $n \in \mathbb{N})$.

Vice versa each $\alpha$ in $\sigma_{2}$ corresponds via $h$ to exactly one decidable subset $B_{\alpha}=\{h(n) \mid \alpha(n)=1\}$ of

\footnotetext{
${ }^{15}$ In functional analysis one expects trouble the soonest.
} 
$\overline{\overline{\sigma_{2}}}$.

Let $j_{\mathcal{C}}$ be the one-to-one correspondence between $\sigma_{2}$ and $\mathcal{C}$ given in 4.2 , where $j_{\mathcal{C}}(\alpha)=\sum_{n \in \mathbb{N}} 2 \alpha(n) \cdot 3^{-n-1}$ for $\alpha$ in $\sigma_{2}$. Let $\alpha$ be in $\sigma_{2}$, write $\alpha_{\mathrm{e}}$ for the element of $\sigma_{2}$ given by $\alpha_{\mathrm{e}}(n)=\alpha(2 n)$ (for all $n \in \mathbb{N}$ ), and $\alpha_{o}$ for the element of $\sigma_{2}$ given by $\alpha_{o}(n)=\alpha(2 n+1)$ (for all $n \in \mathbb{N}$ ). Consider the uniformly continuous function $f_{\text {bar }}$ from $\sigma_{2}$ to $\mathbb{R}^{2}$ given by:

$f_{\text {bar }}(\alpha)=\left(j_{\mathcal{C}}\left(\alpha_{\mathrm{e}}\right), \sup \left(\{0\} \cup\left\{2^{-n} \mid \bar{\alpha}_{o}(n) \in B_{\alpha_{\mathrm{e}}}\right\}\right)\right)$.

A more graphical description: for each $\alpha$ in $\sigma_{2}$ we look at $j_{\mathcal{C}}\left(\alpha_{\mathrm{e}}\right)$ on the $x$-coordinate axis, and we put a dot at $\left(j_{\mathcal{C}}\left(\alpha_{\mathrm{e}}\right), 2^{-m}\right)$ as soon as we encounter the first $m$ such that $\alpha_{o}(m)$ is in $B_{\alpha_{\mathrm{e}}}$. To ensure that we put a dot even if this never occurs we actually put the dot at the $\operatorname{supremum} \sup \left(\{0\} \cup\left\{2^{-n} \mid\right.\right.$ $\left.\left.\bar{\alpha}_{o}(n) \in B_{\alpha_{\mathrm{e}}}\right\}\right)$. The collection of all such 'dots' is the image of $\sigma_{2}$ under $f_{\mathrm{bar}}$.

5.4 We can now prove another theorem very much like theorem 1.3. We use $\sigma_{2}$ instead of $[0,1]$ in this theorem in (2)(c), coming a little closer to the full property Cont III (in fact it would be equivalent to Cont III in the presence of a fifth property stating that the restriction of a 'kontinuous' function is again 'kontinuous').

THEOREM: Within BISH the following statements are equivalent:

(1) The fan theorem FT.

(2) There exists a class of real-valued and $\mathbb{R}^{2}$-valued functions called 'kontinuous' functions such that:

(aa) For all $z \in \mathbb{R}^{2}$ the function $d_{z}$ is kontinuous.

(ab) The function $f_{\text {bar }}$ is kontinuous.

(b) If $f$ and $g$ are kontinuous functions such that $\operatorname{Ran}(f) \subseteq \operatorname{Dom}(g)$, then the composition $g \circ f$ is kontinuous.

(c) If $f$ is a kontinuous function defined on $\sigma_{2}$, then $f$ is uniformly continuous.

(d) The function $x \longmapsto \frac{1}{x}$, defined on $\mathbb{R}^{+}$, is kontinuous.

PROOF: The implication (1) $\Rightarrow(2)$ follows by taking for 'kontinuous' the class of all continuous ${ }^{\mathrm{BRI}}$ real-valued and $\mathbb{R}^{2}$-valued functions. The only nontrivial issue is to show that (2)(d) holds, and this was done in subsection 4.3 in the proof of theorem 1.3. The implication $(2) \Rightarrow(1)$ is trickier but not difficult. Assume (2) and let $B$ be a decidable bar on $\sigma_{2}$. Determine $\gamma=\alpha_{B}$ in $\sigma_{2}$ (see subsection 5.3).

claim $\forall \alpha \in \sigma_{2}\left[f_{\mathrm{bar}}(\alpha) \#\left(j_{\mathcal{C}}(\gamma), 0\right)\right]$.

proof Let $\alpha$ be in $\sigma_{2}$. Since $B$ is a decidable bar on $\sigma_{2}$, we can determine the least $N \in \mathbb{N}$ such that $\bar{\alpha}_{o}(N)$ is in $B$. Determine the natural number $M=h^{-1}\left(\bar{\alpha}_{o}(N)\right.$ ) (see subsection 5.3). We now check on $\alpha_{\mathrm{e}}(M)$. If $\alpha_{\mathrm{e}}(M)=0$ then $\alpha_{\mathrm{e}} \# \gamma$ (because obviously $\gamma(M)=1$ ) and so $j_{\mathcal{C}}\left(\alpha_{\mathrm{e}}\right) \# j_{\mathcal{C}}(\gamma)$ and so $f_{\mathrm{bar}}(\alpha) \#\left(j_{\mathcal{C}}(\gamma), 0\right)$ since $j_{\mathcal{C}}\left(\alpha_{\mathrm{e}}\right)$ is the $x$-coordinate of $f_{\mathrm{bar}}(\alpha)$. If on the other hand $\alpha_{\mathrm{e}}(M)=1$ then $f_{\text {bar }}(\alpha)=\left(j_{\mathcal{C}}\left(\alpha_{\mathrm{e}}\right), 2^{-M}\right)$ and so also $f_{\text {bar }}(\alpha) \#\left(j_{\mathcal{C}}(\gamma), 0\right) \circ$

Now if we take $z=\left(j_{\mathcal{C}}(\gamma), 0\right)$, then $d_{z}$ is a kontinuous function (by (2)(aa)). Also $f_{\text {bar }}$ is kontinuous by (2)(ab). Therefore the composition $d_{z} \circ f_{\text {bar }}$ is a kontinuous function by (2)(b) and by the above claim it goes from $\sigma_{2}$ to $\mathbb{R}^{+}$. The function $x \longmapsto \frac{1}{x}$ is kontinuous by $(2)(\mathrm{d})$, therefore the composition $g$ 
given by $\alpha \stackrel{g}{\longmapsto} \frac{1}{d_{z} \circ f_{\text {bar }}(\alpha)}$ is kontinuous by (2)(b). By (2)(c) $g$ is uniformly continuous and therefore we can determine $N \in \mathbb{N}$ such that for all $\alpha$ in $\sigma_{2}$ we have $g(\alpha)<2^{N}$. So for all $\alpha$ in $\sigma_{2}$ we have $d_{z} \circ f_{\mathrm{bar}}(\alpha)>2^{-N}$.

claim $\forall \beta \in \sigma_{2} \exists n \in \mathbb{N}[n<N \wedge \bar{\beta}(n) \in B]$.

proof Let $\beta$ be in $\sigma_{2}$. Determine $\alpha$ in $\sigma_{2}$ such that $\alpha_{\mathrm{e}}=\gamma$ and $\alpha_{o}=\beta$. Then the $x$-coordinate of $f_{\mathrm{bar}}(\alpha)$ is $j_{\mathcal{C}}(\gamma)$. We had already discovered that $d_{z} \circ f_{\mathrm{bar}}(\alpha)>2^{-N}$, or in other words that $d_{\mathbb{R}^{2}}\left(f_{\text {bar }}(\alpha),\left(j_{\mathcal{C}}(\gamma), 0\right)\right)>2^{-N}$. Since the $x$-coordinate of $f_{\text {bar }}(\alpha)$ is $j_{\mathcal{C}}(\gamma)$ we find that the $y$-coordinate of $f_{\mathrm{bar}}(\alpha)$ is greater than $2^{-N}$. By the definition of $f_{\text {bar }}$ this implies: $\exists n \in \mathbb{N}[n<N \wedge \bar{\beta}(n) \in B] \circ$

So assuming (2) we have shown that an arbitrary decidable bar $B$ on $\sigma_{2}$ contains a finite bar, which amounts to FT

5.5 With the above theorem 5.4 we already hope to convince the reader that amending Cont $\mathbf{I}$ for finding a workable definition of 'continuous function' which reproduces enough of classical mathematics and yet does not imply the fan theorem is not feasible.

Some however might state that $f_{\mathrm{bar}}$ is constructed only for this article, and therefore is not of much interest and might be excluded from the class of 'kontinuous' functions. We have pondered on the possibility of such a reaction. It probably wouldn't help if we argued that $f_{\text {bar }}$ is a very beautiful uniformly continuous function, nor if we argued that other even nicer functions which play a same role as $f_{\text {bar }}$ are likely to show up. We did however find an 'extremely nice' function which already causes enough problems for amending Cont I, although we have been unable to show that 'kontinuity' of this function implies FT. The function yields a counterexample to FT in the context of recursive mathematics. It is in essence the same as the counterexample given in [Kleene\&Vesley1965], but with an added flavour which is useful to us later on. Since the role of recursive mathematics is important in the discussion in section 8 , we feel that this 'extremely nice' function merits presentation in the next section.

5.6 This section's aim was to give some background on possible alternative definitions of 'continuous function'. The most obvious alternative is to drop Cont III as a required property. To maintain the spirit of BISH one then still has to look especially into continuous functions being uniformly continuous near compact subspaces (see subsection 5.1 and section 8 ). This will eventually bring about the same problems, so it is a postponement of trouble. Nonetheless it merits serious consideration, since it provides an equal footing for classical, intuitionistic and recursive mathematics, with transparent definitions. It is a pragmatic approach which can serve as a basis for different general approaches.

Perhaps the so far next best alternative, dropping Cont II as in continuity ${ }^{\mathrm{BIS}}$, can be done without too many problems for elegance and generality. This deserves to be carefully looked at, investigating consequences also in other fields than (linear) analysis. 


\section{A COUnterexample to FT in RECURSive mathematics}

6.1 The search for an 'extremely nice' uniformly continuous function defined on $\sigma_{2}$ which causes similar problems for finding a suitable class of 'kontinuous' functions as $f_{\mathrm{bar}}$ (see 5.3), leads to a counterexample to FT in recursive mathematics which is presented in this section. In essence it is the same as the example given in [Kleene\&Vesley1965], but its specific form here should be of interest.

For this we will indicate a recursive element of $\mathbb{R}^{\mathbb{N}}$ which is recursively apart from all elements of $\mathbb{R}^{\mathbb{N}}$ which have a recursive binary expansion in each coordinate (definitions follow). This revindicates an old question posed by Brouwer in [Brouwer1922], the question whether each real number has a decimal expansion. Again we need some preliminary definitions.

DEFINITION: A real number $x$ is said to be a binary real iff there is an $m \in \mathbb{Z}$ and an $\alpha$ in $\sigma_{2}$ such that $x \equiv_{\mathbb{R}} m+\sum_{n \in \mathbb{N}} \alpha(n) \cdot 2^{-n-1}$. For $x$ in $[0,1]$ we can take $m=0$, so that $x$ in $[0,1]$ is a binary real iff there is an $\alpha$ in $\sigma_{2}$ such that $x \equiv_{\mathbb{R}} \sum_{n \in \mathbb{N}} \alpha(n) \cdot 2^{-n-1}$ and this $\alpha$ is then called a binary expansion of $x$. An $x$ in $\mathbb{R}$ is called a recursive real iff it is equivalent to a recursively defined Cauchy-sequence of rational numbers. An $x$ in $[0,1]$ is called a recursive binary real iff it has a recursively defined binary expansion (this can be easily generalized to $\mathbb{R}$ ). We write $\mathbb{R}_{\text {bin }}$ for the set of all binary reals, and $[0,1]_{\text {bin }}$ for the set of all binary reals in $[0,1]$. Likewise we write $\mathbb{R}_{\text {bin }}^{\mathbb{N}}\left(\right.$ resp. $\left.[0,1]_{\text {bin }}^{\mathbb{N}}\right)$ for the set of all infinite sequences of binary reals (resp. binary reals in $[0,1]$ ). We define the canonical uniformly continuous surjection $k_{\text {bin }}$ from $\sigma_{2}$ to $[0,1]_{\text {bin }}$ as follows:

$k_{\text {bin }}(\alpha) \overline{\bar{D}} \sum_{n \in \mathbb{N}} \alpha(n) \cdot 2^{-n-1}$ (for all $\alpha$ in $\sigma_{2}$ ).

Next let $h$ be the unique bijection from $\mathbb{N}^{2}$ to $\mathbb{N}$ such that $h(n, m)<h(s, t) \Longleftrightarrow(n, m)<_{\text {lexsum }}(s, t)$ where $(n, m)<_{\text {lexsum }}(s, t)$ iff $n+m<s+t \vee(n+m=s+t \wedge n<s)$. We can use $h$ to view each $\alpha$ in $\sigma_{2}$ as an infinite sequence of elements of $\sigma_{2}$. For this we define, for each $n \in \mathbb{N}$ and $\alpha$ in $\sigma_{2}$ the element $\alpha^{[n]}$ of $\sigma_{2}$ as follows:

$\alpha^{[n]}(m) \overline{\bar{D}} \alpha(h(n, m))$ (for all $\left.m \in \mathbb{N}\right)$.

We define a uniformly continuous surjection $k_{\text {bin }}^{\mathbb{N}}$ from $\sigma_{2}$ to $[0,1]_{\text {bin }}^{\mathbb{N}}$ thus:

$k_{\text {bin }}^{\mathbb{N}}(\alpha)(n) \overline{\bar{D}} k_{\text {bin }}\left(\alpha^{[n]}\right)$ (all $\alpha$ in $\sigma_{2}$ and all $n \in \mathbb{N}$ ).

We say that an element $x$ of $\mathbb{R}^{\mathbb{N}}$ is a recursive infinite-real iff $x$ is a recursive sequence of recursive reals. Finally we say that an element $x$ of $[0,1]_{\text {bin }}^{\mathbb{N}}$ is a recursive binary infinite-real iff $x$ is a recursive sequence of recursive binary reals.

REMARK: An element $x$ of $[0,1]_{\text {bin }}^{\mathbb{N}}$ is a recursive binary infinite-real iff there is a recursive $\alpha$ in $\sigma_{2}$ such that $x \equiv_{\mathbb{R}^{\mathbb{N}}} k_{\text {bin }}^{\mathbb{N}}(\alpha)$. This may be taken as the formal definition if necessary.

6.2 The uniformly continuous function $k_{\text {bin }}^{\mathbb{N}}$ is the 'extremely nice' function promised at the end of the previous section. The following theorem will eventually show that $k_{\text {bin }}^{\mathbb{N}}$ causes problems similar to the problems caused by $f_{\text {bar }}$ in the previous section. 
ThEorem: There is a recursive infinite-real $\beta_{\# \text { bin }}$ in $[0,1]^{\mathbb{N}}$ which is recursively apart from each recursive binary infinite-real in $[0,1]_{\text {bin }}^{\mathbb{N}}$.

The proof of this theorem involves some technicalities and is postponed until subsection 10.1 so that we can directly focus on the interesting consequences of the theorem. Mathematicians experienced in constructive / intuitionistic mathematics will have no trouble to deduce from theorem 6.2 the following corollary:

COROLlary: (to theorem 6.2) There is a decidable subset $B$ of $\overline{\bar{\sigma}}_{2}$ such that $B$ is a bar on $\sigma_{2 \text { ReC }}$ and yet $B$ contains no finite bar on $\sigma_{2 \mathrm{REC}}$. In other words: the fan theorem FT fails in recursive mathematics.

PROOF: We use lemma 4.1. Let $\beta_{\# \text { bin }}$ be as in the theorem, let $f$ be given by: $f(x) \overline{\bar{D}} d_{\mathbb{R}^{\mathbb{N}}}\left(k_{\text {bin }}^{\mathbb{N}}(x), \beta_{\# \text { bin }}\right)$ which is a uniformly continuous function from $\left(\sigma_{2}, d_{\omega}\right)$ to $\left(\mathbb{R}, d_{\mathbb{R}}\right)$. By lemma 4.1 there is a decidable subset $B$ of $\overline{\bar{\sigma}}_{2}$ such that:

(i) $\forall \alpha \in \sigma_{2} \forall n \in \mathbb{N}\left[\bar{\alpha}(n) \in B \rightarrow f(\alpha)>2^{-n}\right]$

(ii) $\forall \alpha \in \sigma_{2}[f(\alpha)>0 \rightarrow \exists m \in \mathbb{N}[\bar{\alpha}(m) \in B]]$

Clearly this $B$ is the desired decidable bar on $\sigma_{2 \mathrm{REC}}$ containing no finite bar on $\sigma_{2 \mathrm{REC}}$ (since the image of $\sigma_{2 \text { REC }}$ under $k_{\text {bin }}^{\mathbb{N}}$ is dense in $[0,1]_{\text {bin }}^{\mathbb{N}}$ )

6.3 But the main reason for presenting theorem 6.2 is because it reflects on our analysis concerning the definition of 'continuous function' in BISH. This is best spelled out with another theorem quite like theorem 5.4:

THEOREM: It is not possible to prove within BISH that there exists a class of real-valued and infinitereal-valued functions called 'kontinuous' functions such that:

(aa) For all $x \in \mathbb{R}^{\mathbb{N}}$ the function $d_{x}$ given by $y \stackrel{d_{x}}{\longmapsto} d_{\mathbb{R}^{\mathbb{N}}}(x, y)$ is kontinuous.

(ab) The function $k_{\text {bin }}^{\mathbb{N}}$ is kontinuous.

(b) If $f$ and $g$ are kontinuous functions such that $\operatorname{Ran}(f) \subseteq \operatorname{Dom}(g)$, then the composition $g \circ f$ is kontinuous.

(c) If $f$ is a kontinuous function defined on $\sigma_{2}$, then $f$ is uniformly continuous.

(d) The function $x \longmapsto \frac{1}{x}$, defined on $\mathbb{R}^{+}$, is kontinuous.

PROOF: The idea of the proof is of course the same as in the proof of theorem 5.4. Suppose we can prove within BISH the existence of a class of real-valued and infinite-real-valued functions called 'kontinuous' functions which satisfies (aa)-(d) above. Then this class exists also in recursive mathematics. Now focus on recursive mathematics, and consider the recursive infinite-real $\beta_{\# \text { bin }}$ in $[0,1]^{\mathbb{N}}$ given by theorem 6.2 , such that $\beta_{\# \text { bin }}$ is recursively apart from each recursive binary infinite-real in $[0,1]_{\text {bin }}^{\mathbb{N}}$. Since $[0,1]_{\text {bin }}^{\mathbb{N}}$ is dense in $[0,1]^{\mathbb{N}}$, we have that $\inf \left(\left\{d_{\beta_{\# \text { bin }}}(x) \mid x \in[0,1]_{\text {bin }}^{\mathbb{N}}\right\}\right)=0$, but at the same time the function $d_{\beta_{\# \text { bin }}}$ restricted to $[0,1]_{\text {bin }}^{\mathbb{N}}$ has for its range a subset of $\mathbb{R}^{+}$. So finally we consider the composition of the three kontinuous functions $k_{\text {bin }}^{\mathbb{N}}, d_{\beta}$ \#bin and $x \longmapsto \frac{1}{x}$ (kontinuous by (ab), (aa) and (d)). This 
is the function $k$ given by $k(\alpha)=\frac{1}{d_{\beta} \# \text { bin }}(\alpha)$. By (b) $k$ is kontinuous, and by (c) $k$ is uniformly continuous on $\sigma_{2}$. However this is impossible, since for each $N \in \mathbb{N}$ we can find an $\alpha$ in $\sigma_{2}$ REC such that $k(\alpha)>N$. Contradiction, therefore the theorem holds

We hope that in the light of section 5 this theorem will convince the remaining few that the amending of Cont I is not a feasible option for coming to a BISH-definition of 'continuous function'. The proof of theorem 6.2 omitted in this section will be given in the appendix which is the final section.

\section{Reality, Determinism and Church's Thesis}

7.1 The fact that FT does not hold in RUSS is well-known (in the next section some mathematical foundational discussion about this is offered). In this section we look at a theorem proved in [Bridges\&Richman1987], which shows a little more than the failure of FT in RUss. The theorem carries in it the possibility for an experiment in the real world, which could give statistical evidence on whether the real world is 'deterministic' or not. This is an old debate, unlikely to be easily resolved. This section aims at providing a mathematical perspective, which might help clarify the debate. Even with this mathematical perspective, the statistical setting of the experiment is complicated, and touches on the foundations of probability theory. It remains the question, therefore, whether the old debate can be resolved at all. The main relevance of this section is for our foundational discussion: it clarifies the different perspectives on reality of RUSS on one side, and CLASS and INT on the other.

The section is built around a constructive-mathematician's analysis of what we mean with 'the real world is deterministic'. Determinism is a much studied 'doctrine' in philosophy, and there are more than one philosophical 'definitions' (read: interpretations) of the word 'determinism'. Since whole books have been written on the subject (see e.g. [Earman1986]), we cannot possibly reproduce all the possible philosophical positions here, nor do we wish to do so. Our main interest devolves from the interpretations of determinism that modern physics tries to prove or disprove. These efforts have received quite some attention, both in philosophy and in physics.

The most common interpretation of determinism is an ontological one, called Laplacian determinism. The 'definition' of Laplacian determinism is commonly phrased thus: 'the instantaneous state of the world at any time uniquely determines the state of the world at any later time'. Basically the idea is that there is no freedom at all in the temporal evolution of the world: from a given beginstate, everything follows 'mechanically'. (This does not necessarily mean that the world is then predictable, since it may then be and most likely is! fundamentally impossible to obtain the necessary 'data' and the necessary 'machine' or 'mechanical computations' to actually predict in advance what is going to happen.).

Unfortunately, there is no widely accepted sharper definition of Laplacian determinism. In the author's eyes this has led to a number of mathematically vague attempts to prove or disprove the statement 'the real world is deterministic' in modern physics. One sees indeed that various attempts are not disputed for their physical outcome, but for whether from this outcome Laplacian determinism can be conclusively proven or dismissed. Now fortunately, the frameworks developed in constructive mathematics offer a sharp mathematical interpretation of Laplacian determinism. In fact it is a very consequent carrying through of Church's Thesis. Church's Thesis has been related to determinism by many authors, even 
as radically as we propose (see [FoM2001]). We will see in this section that this leads to an interesting physical experiment, to (dis)prove determinacy of the real world.

However, we also note in advance that our preferred interpretation is more or less dismissed in [Earman1986] (chapter VI: Determinism, mechanism and effective computability) as being 'simply not true'. It should be noted that in [Earman1986] it is taken for granted, without explanation, that classical mathematics is the only truth available when it comes to describing the real world. This tacit and unexplained assumption underlies many discussions in modern physics, and especially the one on determinism. We cannot therefore emphasize enough that the foundational issues of modern mathematics are of vital importance to the foundational issues of modern physics (and vice versa).

7.2 Like some other authors, we propose that Laplacian determinism be seen in the light of constructive mathematics and Church's Thesis. This means amongst other things that infinite sequences (of natural numbers; a real number is then given by such an infinite sequence) are never 'finished', instead we see them developing in the course of time. Now a very consequent, therefore elegant interpretation of Laplacian determinism runs as follows. Suppose that there is in the real world a developing-infinite sequence of natural numbers, say $\alpha$. Then how to interpret the statement that this sequence is 'uniquely determined' by the state of the world at time zero? At time zero we can have at most finite information since according to our constructive viewpoint, infinity is never attained. So this finite information about $\alpha$ supposedly enables us to 'uniquely determine' $\alpha$ in its course of time. It is now hard to see another interpretation of this last statement, than the one given by Church's Thesis, namely that this finite information must be a (Turing-)algorithm that we can use to compute $\alpha(n)$ for any $n \in \mathbb{N}$.

With classical logic and omniscience, the previous can be stated thus: 'for every (potentially infinite) sequence of numbers $\left(a_{n}\right)_{n \in \mathbb{N}}$ taken from reality there is a recursive algorithm $\alpha$ such that $\alpha(n)=a_{n}$ for

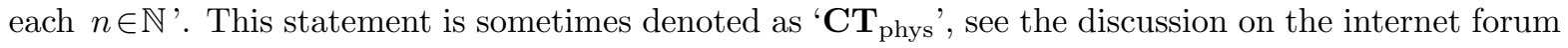
'Foundations of Mathematics' ([FoM2001]). As stated in the introduction, this classical omniscient interpretation is easily seen to fail in real life. Therefore we adopt the constructive viewpoint. The statement 'the real world is deterministic' can then best be interpreted as: 'a (potentially infinite) sequence of numbers $\left(a_{n}\right)_{n \in \mathbb{N}}$ taken from reality cannot be apart from every recursive algorithm $\alpha$ (in symbols: $\left.\neg \forall \alpha \in \sigma_{\omega \mathrm{REC}} \exists n \in \mathbb{N}\left[\alpha(n) \neq a_{n}\right]\right)^{\prime}$.

We adopt this interpretation in the rest of the paper. We do not have the time and space (...) here to extensively defend this interpretation, since we are primarily concerned with the foundations of mathematics. We will however point out the connection between these foundations and our interpretation of determinism. Also there is a direct connection with physics.

For under the above interpretation of Laplacian determinism, we hold that there is a physical experiment which can give statistical evidence whether the real world is deterministic or not. The experiment consists 'simply' of testing whether an arbitrary sequence taken from the physical reality is given by a recursive rule or not (so even if one disagrees with the linking of determinism to Church's Thesis, one at least has an experiment which might decide whether the physical world is capable of producing a non-recursive infinite sequence). For this experiment, we first introduce some well-known mathematical notions in a simplified form. Measure theory is a mathematical discipline which introduces the concept of 'size' also to infinite sets, in such a way that the measure of the union of two disjunct measurable sets is the sum of the measures of the two sets involved. One basic motivation for this is the theory of integration. A restricted class of functions which are defined 'almost everywhere' on e.g. [0,1] can 
be integrated. 'Almost everywhere' means that the subset on which such a function is not defined, has measure 0. There are infinite subsets of $[0,1]$ which have measure 0 , such as $\mathbb{Q} \cap[0,1]$, but also the uncountable Cantor set $\mathcal{C}$. On the other hand, the measure of any real interval $[\alpha, \beta]$ is simply its length $\beta-\alpha$ (it doesn't matter whether the endpoints of the interval are included or not, since they have measure 0 ).

However, even in CLASS not every subset of $[0,1]$ is measurable. This is one of the issues that sparked foundational disagreements in the beginning of the twentieth century. The construction in CLASS of a non-measurable subset of $[0,1]$ by Vitali in 1905 made essential use of the newfound classical axiom of choice, which arose out of Cantor's work on set theory. In constructive mathematics already a countable union of disjoint intervals need not be measurable, since we cannot always determine the supremum of the partial sums of the lengths of the intervals involved.

7.3 Linking measure theory to probability theory, the measure of a subset of $[0,1]$ can be seen as the likelihood that an arbitrary element of $[0,1]$ is in that subset. Now in [Bridges\&Richman1987] (Ch. 3, thm. 4.1) it is shown how to construct, for every $n \in \mathbb{N}$, a bar $R_{n}$ on $[0,1]_{\mathrm{REC}}$ (in fact a countable sequence of intervals such that the union contains $\left.[0,1]_{\mathrm{REC}}\right)$ such that this bar in CLASS has measure less than $2^{-n}$. Constructively we cannot arrive at the same conclusion, since we cannot assert that these bars are measurable ${ }^{16}$. This is due as indicated to the constructive difficulty in determining the supremum of the partial sums of the lengths of the intervals involved. Nonetheless it is easily seen that for each $n \in \mathbb{N}$, the partial sums of the lengths of the intervals involved in $R_{n}$ can never exceed $2^{-n}$.

Therefore we can still say that classically and intuitionistically the likelihood of an arbitrary element of $[0,1]$ being in $R_{n}$ is less than $2^{-n}$. But clearly, in Russ this probability is 1 . This is the basis for our real-world experiment. We conduct this experiment in the usual scientific way, with two hypotheses $\mathrm{H}_{0}$ and $\mathrm{H}_{1}$, and an uncertainty parameter $\alpha_{0}$. The aim is to disprove $\mathrm{H}_{0}$, by arriving at an experimental event which under the assumption of $\mathrm{H}_{0}$ will take place with probability less than $\alpha_{0}$. As a result we then accept $\mathrm{H}_{1}$. This procedure is not symmetrical and it can be worthwile to switch $\mathrm{H}_{0}$ and $\mathrm{H}_{1}$ (which is done most easily by introducing a reciprocal uncertainty parameter $\alpha_{1}$ ). For most experiments $\alpha_{0}$ is taken somewhere in the interval $\left[10^{-3}, 10^{-2}\right]$, but of course we can afford to be liberal, and there is much at stake, so we suggest for this experiment $\alpha_{0}=2^{-40} \approx 10^{-12}$.

We start with the easiest experimental setting by defining our hypotheses as follows:

$\mathbf{H}_{0}$ The world is infinite in time and non-deterministic, therefore the recursive sequences form a negligible subset of the set of all infinite sequences.

$\mathbf{H}_{1}$ The world is infinite in time yet deterministic, therefore there is no sequence which is not given by a recursive algorithm.

We concentrate on $\mathbf{H}_{0}$ and $\mathbf{H}_{1}$, and disregard the finitistic point of view. ${ }^{17}$ Now for the corresponding experiment. We take some natural fluctuating phenomenon (atmospheric pressure, oceanic waterflow, temperature?) which gives us fluctuating data which are measured with high precision ${ }^{18}$, say every millisecond. It is easy to convert this growing stream of data to a fast-growing 'infinite' sequence of

\footnotetext{
${ }^{16}$ In fact there is a constructive theorem saying that every measurable bar on $[0,1]$ has measure 1.

${ }^{17}$ If the world is bounded in time in such a way that the experiment comes to an end before giving a result, then everything ends and there is no problem. If not, then there is no problem either.

${ }^{18}$ The high-precision is needed to have the natural fluctuation numerically expressed.
} 
decimals, to obtain a real-time decimal expansion (more precise: approximation) of an element $\beta$ of $[0,1]$. At the same time we start constructing the recursive bar $R_{40}$, which can be enumerated as a series of open intervals $\left(I_{m}\right)_{m \in \mathbb{N}}$. The experiment finally consists of nothing more than checking for each $m \in \mathbb{N}$, whether $\beta$ is in $I_{m} . \mathbf{H}_{0}$ states that the probability of this occurring for any $m \in \mathbb{N}$ is less than $10^{-12}$. Therefore if for some $m \in \mathbb{N}$ we do indeed arrive at the conclusion that $\beta$ is in $I_{m}$, then the world is deterministic with overwhelming probability. On the other hand, if the world is deterministic then this will eventually be discovered by this experiment, if we wait long enough ${ }^{19}$ !

7.4 As said, the experiment is not symmetrical w.r.t. the hypotheses above. It would be worthwile to consider $\alpha_{1}=2^{-40}$ and see if we can define a reciprocal event in the above experiment which under assumption of $\mathbf{H}_{1}$ will take place with probability less than $\alpha_{1}$. For this we would have to be able for each $n \in \mathbb{N}$, to tell something about the probability that for an arbitrary recursive element $\gamma$ of $[0,1]$, we have to wait longer than $n$ before discovering an $m \in \mathbb{N}$ for which $\gamma$ is in $I_{m}$. We frankly don't know whether it is possible to pin down these probabilities. It is too complicated for us, and for the context of this paper, but hopefully somebody better-versed in probability and recursion theory will take up this challenge. ${ }^{20}$

As it stands described above, if the world is deterministic the experiment gives a possibility for RUSS to prove that CLASS and INT are unrealistic. But we don't know how long we must wait for such a result, since even under $\mathbf{H}_{1}$ the algorithm which computes our natural phenomenon is still unknown to us. Now if the definition of a reciprocal event is possible, then one has a reciprocal statistical way of proving RUSS to be unrealistic (depending on the outcome of course). It seems to the author that this is interesting enough to look into. One must not be surprised of course, in the event that such a reciprocal event can be defined, when it turns out that the numerical complexity of such an experiment will elude our limited computational powers. Therefore the finitistic standpoint cannot be completely ignored.

Since in any way this experiment has not yet been conducted, the author is free to believe in his own bias, namely that the world is non-deterministic. This bias will be seen to influence the discussion in the next section. Notice that the next section is written in a more familiar mathematical setting, almost as if this section did not exist. Yet an outcome of an experiment as described above would impact heavily, if one is concerned with how accurately mathematics reflects reality. ${ }^{21}$

\footnotetext{
${ }^{19}$ This is some form of Markov's Principle of course.

${ }^{20}$ The author sees a connection with Benford's law and the fundamental problem of 'picking a natural number at random' (see [Hill1996], [DeFinetti1972] and [DeFinetti1974]). For suppose $\mathbf{H}_{1}$ holds, than (with classical logic) every sequence (finite or potentially infinite) is given by a partial recursive algorithm, which can be seen as an element of $I(\mathbb{N}, \mathbb{N})$. Consider the probability that for a given $e$ in $I(\mathbb{N}, \mathbb{N})$ the sequence $\beta$ in our experiment runs through an $I_{m}$ which is derived by letting $e$ compute its first so many values. Many mathematicians would state that this probability cannot be equal for all $e$, since by the hypothesis the sum of all these probabilities equals 1 . Therefore as $e$ goes to infinity, the probability of $e$ being the first number to yield an $I_{m}$ containing $\beta$ goes to zero, in such a way that the sum of all these probabilities equals 1. But the distribution of these probabilities in the real physical world is unknown. This is a phenomenon which holds for the set $\mathbb{N}$ as well: what is the probability of an arbitrary natural number turning up in reality? In a follow-up paper we intend to give an alternative explanation of Benford's law, which opens up a new direction how to tackle these questions. In [DeFinetti1974] a fundamental different position is stated: countable additivity of probabilities is rejected, equal (zero) probability of natural numbers then becomes possible with the sum of all probabilities still equal to 1.

${ }^{21}$ Even without outcome, the experiment has the merit of putting the debate 'deterministic or non-deterministic' in a blunt and therefore clear perspective.
} 


\section{Reuniting THE ANTIPODES?}

8.1 In this section we at times adopt a slightly provocative point of view, which we hope will not antagonize. Our aim is to stimulate discussion in the realm of constructive mathematics, especially foundational matters. We certainly do not claim to have final wisdom. And our standpoint will be recognizably biased.

We have seen that the spirit of BISH - constructive mathematics following classical mathematics as much as possible - is intimately connected to the fan theorem FT. From theorem 1.3 the question arises whether the scope and/or focus of BISH as it stands today is any different from the scope and/or focus determined by BISH + FT. In other words: is the intuition behind BISH any different from the intuition behind FT?

We think not. We are so bold to suggest that a historic problem of communication is the main cause of the separation between BISH and FT. Bishop clearly thought the fan theorem to be a mystic element of intuitionism. His understandable reaction was not to accept it, however we doubt that he did this with a clear view of the relationship between the fan theorem and the theory of continuous functions, as put forward in theorem 1.3. We deduce this from writings of Bishop himself.

8.2 In an unfinished never-published preprint called 'The neat category of stratified spaces' Bishop admits the problem that one cannot prove within BISH that the composition of two continuous ${ }^{\mathrm{BIS}}$ functions is again continuous ${ }^{\mathrm{BIS}}$. In this preprint he puts forward the solution of continuity ${ }^{\mathrm{BRI}}$ (adopted in [Bridges1979]) as the definitive and final constructive definition of continuity. Theorem and corollary 1.3 show that this approach yields an equivalence between the fan theorem and the continuity ${ }^{\mathrm{BRI}}$ of the beautiful function $x \longmapsto \frac{1}{x}$. The unpublished preprint in our eyes therefore reflects Bishop's unawareness of the relationship between the fan theorem and the theory of continuous functions, as in theorem 1.3.

Brouwer, on the other hand, formulated the fan theorem rather late in his intuitionistic career. At that time he had accumulated a vast knowledge and intuition of topology. His treatment of the fan theorem is exceptional: he proves it using a stronger axiom of bar induction ${ }^{22}$. In hindsight we think that Brouwer at least suspected relationships like in theorem 1.3. We deem it extremely likely that Brouwer knew or felt that an elegant intuitionistic theory was not possible without the fan theorem. For a topologist like Brouwer, 'elegant' would almost surely have to imply that the composition of two continuous functions is again continuous, and that a continuous function is indeed uniformly continuous on a compact space.

However, simply adopting the fan theorem in itself as an axiom might strike one as being rather opportunistic (this reflects some of Bishop's resentment of the fan theorem). So if our little 'psychological analysis' is correct, which it probably isn't!, Brouwer was faced with the problem of motivating the fan theorem in a fundamental way, transcending possible opportunistic motives. Personally we think that his solution to this challenge: bar induction (or rather 'Brouwer's Thesis' as presented later in this section), is a stroke of pure genius.

But quite understandably, Brouwer never motivated the fan theorem in opportunistic terms. The drawback of this has been that the vast topological backing of the fan theorem remained largely in

${ }^{22}$ To be more precise he formulated what [Veldman1981] calls 'Brouwer's Thesis'. We investigate this further on. 
Brouwer's head ${ }^{23}$. Therefore to the analyst Bishop the theoretical edifice leading to the fan theorem probably lacked the conviction of practice, which the topologist Brouwer had in abundance.

Brouwer's style of writing probably wasn't much help either. Still Bishop could have consulted [Kleene\&Vesley1965] to see that the fan theorem isn't mystic at all (and neither is CP). Once again an explanation for Bishop's non-adoption of [Kleene\&Vesley1965] might lie in the difference of language and style. Bishop wanted to do mathematics, practical, down-to-earth. And although he was convinced of Brouwer's view that classical mathematics lives in a dream world ('lacks numerical meaning' according to Bishop), he was strongly against the formal and foundational setting of intuitionism at that time, which had attracted mainly logicians and philosophers.

8.3 So Bishop and more so his followers, notably Bridges and Richman (see [Bridges\&Richman1987] and many other publications), set out on another mission: to reunite the antipodes ${ }^{24}$. In our humble opinion they both succeeded and failed. Successful they have been with respect to changing the focus of constructive mathematics back to analysis (from logic and philosophy). The author is impressed with Bishop's achievement in this sense, since in our view any true mathematics should primarily offer mathematical challenges, and not focus mainly on the meta-mathematical part. By not adopting $\mathbf{C P}$, results of BISH can easily be used by both intuitionistic and classical mathematics, thus bringing closer the antipodes.

Also, results in BISH hold in recursive mathematics. Therefore some see BISH as a generalization of classical, intuitionistic and recursive mathematics, disregarding philosophical issues. But BISH in our eyes does not take up a neutral position with respect to RUSS, especially since there are many continuous functions in recursive mathematics which are not continuous ${ }^{\mathrm{BIS}}$ or continuous ${ }^{\mathrm{BRI}}$. Therefore these functions are not studied as continuous functions in BISH. The only way to become a common theory for intuitionism, classical and recursive mathematics is to change the spirit of BISH, redirecting BISH more towards recursive mathematics by giving this branch more serious attention. In terms of the definition of 'continuous function' this means dropping Cont III from the list of properties that a continuous function should possess. In other words, this means adopting definition 3.1 and live with the possible existence of continuous functions on $[0,1]$ which are not uniformly continuous.

It then seems wise to develop a general theory of continuous functions, as well as a general theory of uniformly continuous functions. In this setting, the functions for which we know that they are uniformly continuous near each compact subspace (let's call these functions continuous ${ }^{\mathrm{BI}+}$ ) do not automatically form a nice subclass (again, this would imply FT). But one can formulate conditions under which the composition of two continuous ${ }^{\mathrm{BI}+}$ functions is again continuous ${ }^{\mathrm{BI}+}$, as in lemma 3.3. Then all three antipodes might benefit from this approach in an equal manner. In the light of the previous section, the obvious advantage is that such a mathematics will reflect reality whether reality is deterministic or not. As long as we cannot settle that question, it won't be harmful to keep the options open. So we plead for some adaptation of BISH, see the discussion later on.

In the same vein, one should then develop RUSS to the point where RUSS takes into consideration that even if the world is deterministic, in reality we still will not be supplied with much knowledge about the algorithms producing our 'infinite' sequences. As stated earlier, we think it possible to model this

\footnotetext{
${ }^{23}$ It must be said that a large part of Brouwer's unpublished writings was lost, quite possibly his notes would have shed a different light altogether on this discussion.

${ }^{24}$ Thanks to Peter Schuster for this terminology.
} 
mathematically by introducing two players, player I and player II. Player I hands out infinite sequences to player II, but in principle does not reveal what algorithm is behind the sequences, although sometimes in her benevolence she gives crumbs of knowledge to player II... We think that in such a model CP is still consistent. But FT still fails, and of course player II can do the same experiment as described in the previous section to find this out.

From the previous section there is one question which in this context begs asking. We have seen that there are bars on $\sigma_{2 \mathrm{REC}}$ with arbitrary small classical measure. But these bars are not measurable in a constructive sense. We saw that constructively measurable bars on $[0,1]$ all have measure 1 . Now could it be that an adaptation of FT which limits itself to measurable bars, would also hold in RUss? The following proposition shows that this is not the case.

PROPOSITION: There is a measurable bar on $\sigma_{2 \mathrm{REC}}$ which does not contain a finite bar on $\sigma_{2 \mathrm{REC}}$.

PROOF: It suffices to consider the bar $B$ given in the proof of corollary 6.2. The standard measure on $\sigma_{2}$ is determined by the measure of the basic open sets, given as follows: $\mu\left(\left\{\alpha \in \sigma_{2} \mid \bar{\alpha}(n)=a\right\}\right)=2^{-n}$, for $a$ in $\bar{\sigma}_{2}, \lg (a)=n$. It is not difficult to see that $B$ (more precise: $\left\{\beta \in \sigma_{2} \mid \exists n \in \mathbb{N}[\bar{\beta}(n) \in B]\right\}$ ) is measurable and has measure 1 , since $\left\{\beta \in \sigma_{2} \mid \exists n \in \mathbb{N}[\bar{\beta}(n) \in B]\right\}$ is the inverse image under $k_{\text {bin }}^{\mathbb{N}}$ of the open set $C=\left\{\alpha \in[0,1]^{\mathbb{N}} \mid \alpha \# \beta_{\# \text { bin }}\right\}$. By the proof of corollary 6.2 we have that $B$ does not contain a finite bar on $\sigma_{2 \mathrm{REC}}$

8.4 Back to our main theme: the position of BISH. It is safe to assume that Bishop didn't really believe that RUSS is the most realistic mathematics. The fact that BISH has consequently sought to maintain Cont III shows that the heart of BISH lies with CLASS and INT. In bringing these antipodes together we feel BISH has fallen short. For we think that if one wishes to reduplicate enough classical results, which was at least part of the thrust of BISH, then dropping the fan theorem is too radical a move. It is present both in intuitionistic and classical mathematics, whereas its formal absence causes all sorts of problems for elegance and generality, testified to by theorem 1.3. Therefore in BISH one comes across constructions and make-do definitions to avoid a fan theorem to which both the antipodes agree ${ }^{25}$. As a consequence, it is at times not very attractive for intuitionism or classical mathematics to really adopt the BISH approach.

8.5 Asked by one of our critics for an illustration of this unattractiveness, we look at the classical and intuitionistic homeomorphism $h$ from $\mathbb{R}$ to $\mathbb{R}^{+}$given by:

$h(x) \overline{\bar{D}} \begin{cases}\frac{1}{2-x} & \text { for } x \leq 1 \\ x & \text { for } x \geq 1\end{cases}$

It should be noted that under current definitions in BISH $h$ is NOT a homeomorphism (and the continuity ${ }^{\mathrm{BRI}}$ of $h$ is again equivalent to FT). Related to this is the fact that in BISH the space $\mathbb{R}^{+}$ is NOT locally compact. In BISH a metric space $(X, d)$ is 'locally compact' iff every $d$-bounded subset of $(X, d)$ is contained in a compact subspace of $(X, d)$ (this implies metrical completeness). It is not a topological notion, by which we mean invariant under homeomorphisms (see the appendix). It does not correspond to the classical and intuitionistic notion, which amounts to the usual approach to

\footnotetext{
${ }^{25}$ Moreover the fan theorem in our opinion underlies the intuition behind BISH as well, as explained before.
} 
'local' properties in topology ${ }^{26}$, namely: a topological space $(X, \mathcal{T})$ is locally compact iff for every $x$ in $X$ and every open $U \ni x$ there is a compact neighborhood $W \subseteq U$ of $x .{ }^{27}$ The current BISH definition of 'locally compact' might lead to confusion, especially considering the claim that every BISH result is a classical result. Take for instance the following BISH variant of the Tietze extension theorem ([Bishop\&Bridges1985]):

theorem: (only in BISH!) Let $(A, d)$ be a locally compact subspace of a metric space $(X, d)$. Let $f$ be a continuous function from $(A, d)$ to $\left([0,1], d_{\mathbb{R}}\right)$. Then there is a continuous extension of $f$ to $(X, d)$.

Notice that without translation to the not-so-well-known BISH definition of 'locally compact' the theorem is both classically and intuitionistically false. The BISH definition of 'locally compact' and 'homeomorphism' is partly tied in with the problems arising out of theorem 1.3. We think a better definition is obtained by defining a metric space to be locally compact iff it has a one-point compact extension. We will show in the appendix that this amounts to the usual definition in all three antipodes (see 10.2). However this does mean trouble for BISH in relation to Cont II, or Cont III, for continuity ${ }^{\mathrm{BIS}}$ and continuity ${ }^{\mathrm{BRI}}$.

Interestingly, with the usual definition of 'continuous function' (the one we adopt), we can prove in BISH the Dugundji Extension Theorem, which is stronger than the Tietze extension theorem. We formulate a convenient version of the constructive Dugundji theorem proved in [Waaldijk1996] ${ }^{28}$ :

Theorem: Let $(A, d)$ be a complete and located subspace of a metric space $(X, d)$. Let $f$ be a continuous function from $(A, d)$ to $\left(L, d_{L}\right)$, a complete and locally convex linear space. Then there is a continuous extension $\tilde{f}$ of $f$ to $(X, d)$, such that for all $x$ in $X$ the image $\tilde{f}(x)$ is in the closure of the convex hull of $f(A)$.

The problem with this theorem for BISH is that (so far) we have not discovered a general way of proving within BISH that the extension is continuous ${ }^{\mathrm{BIS}}$ or continuous $^{\mathrm{BRI}}$ if the original function is continuous ${ }^{\mathrm{BIS}}$ respectively continuous ${ }^{\mathrm{BRI}}$. But this problem is non-existent in INT, in CLASS and even in RUSS ${ }^{29}$, so we think the extension theorem above will be preferred by intuitionistic, classical and recursive mathematicians alike. This ends our illustration.

8.6 Recapitulating, a drawback in Bishop's approach is that the common ground of intuitionism and classical mathematics is greater than one would suspect from the results of BISH. So if one would care to reunite these antipodes, it can be done in a much more powerful way. CLASS and INT are much closer to each other, axiomatically speaking, than to RUSs. There is a philosophical motivation for this, namely the earlier mentioned argument that CLASS and INT describe a non-deterministic world, whereas RUSS describes a deterministic world. For the rest of the discussion in this section we focus on the axiomatical differences and similarities. The eccentric position of RUSS as compared to CLASS and INT then comes to the fore naturally.

\footnotetext{
${ }^{26}$ See [Waaldijk1996] for a treatment of local properties in intuitionistic topology.

${ }^{27}$ In INT it seems wise to add the condition that $(X, \mathcal{T})$ be spreadlike.

${ }^{28} \mathrm{~A}$ stronger version is available, but its formulation needs several definitions which would be too troublesome here.

${ }^{29}$ Provided one isn't interested in uniform continuity of the resulting function near compact subspaces.
} 
8.7 Therefore we work to reunite the antipodes CLASS and INT at their formal intersection which 'contains' $\mathbf{B I}_{\mathbf{D}}$. The intersection also contains the Lindelöf property for complete metric spaces and a contrapositive form of Baire's category theorem (intuitionistically derived from $\mathbf{C P}$ ). We now go into this in greater detail, and apologize beforehand for what will seem to some a myriad of axioms. The result will be a plea for four 'axiomatic systems', three of which are currently not basking in attention. The first axiomatic system will in essence be good old BISH. The second will be a formal intersection of all three antipodes, with the addition of MP. The third will be a formal intersection of CLASS and INT, with the addition of MP. The last axiomatic system that we will plead for is traditional intuitionistism $\mathrm{INT}^{30}$, also with the addition of MP. Other possible systems are left to the reader.

8.8 Remember that we took the trouble of repeating the intuitionistic axioms of continuous choice $\mathbf{A C}_{11}$ and the weaker $\mathbf{A C}_{10}$. These axioms conflict with classical mathematics, but there is a very important consequence of $\mathbf{A C}_{10}$ which also holds, mutatis mutandis, in CLASS and RUSs. We formulate this consequence as an axiom called BDD (bar decidable descent). But we first need a definition:

Definition: Let $B$ and $C$ be two bars on $\sigma_{\omega}$, then $B$ descends from $C$ iff for all $c$ in $C$ there is a $b$ in $B$ such that $b \sqsubseteq c$.

BDD Every bar on $\sigma_{\omega}$ descends from a decidable bar on $\sigma_{\omega}$.

REMARK: Notice that $\mathbf{A} \mathbf{C}_{10}$ is equivalent to the combination of $\mathbf{C P}, \mathbf{B D D}$ and $\mathbf{A} \mathbf{C}_{00}$ (proof in section 10, where we also prove that BDD holds in Russ). For elegance we introduce the concept of a 'thin' bar, meaning a bar without redundancy, in the next definition and lemma.

Definition: A bar $B$ on $\sigma_{\omega}$ is called thin iff for all $b$ in $B$ and all $a$ in $\overline{\bar{\sigma}}_{\omega}$ we have: $a \sqsubset b \rightarrow a \notin B$.

LEMMA: Every decidable bar on $\sigma_{\omega}$ contains a decidable thin bar.

PRoof: Let $B$ be a decidable bar on $\sigma_{\omega}$. Consider the bar $C$ given by: $C=\left\{b \in B \mid \forall a \in \overline{\bar{\sigma}}_{\omega}[a \sqsubset c \rightarrow\right.$ $a \notin B]\}$. It is easy to check that $C$ is a thin bar and a decidable subset of $B$

COROLLARY: BDD implies that every bar on $\sigma_{\omega}$ descends from a decidable thin bar.

Call a metric space spreadlike iff it is the continuous image of $\left(\sigma_{\omega}, d_{\omega}\right)$, then it is a well-known observation that every complete metric space is spreadlike. From BDD it follows that every spreadlike metric space is Lindelöf (every open cover admits an enumerable refinement consisting of basic open sets). From this Lindelöf property it follows that every open cover of a spreadlike metric space has a subordinate partition of unity. This in turn gives rise to a number of important theorems concerning continuous functions, such as the Michael Selection Theorem (see [Waaldijk1996]). Of course, combining $\mathbf{B I}_{\mathbf{D}}$ with $\mathbf{B D D}$ one gets the so called principle of monotone bar induction, usually denoted $\mathbf{B I}_{\mathbf{M}}$. Also with BDD one can strengthen FT to the statement that every bar on $\sigma_{2}$ contains a finite bar, dropping the precondition of decidability. This leads to the following important proposition:

PROposition: From BDD and FT it follows that every open cover of a compact space has a finite

\footnotetext{
${ }^{30}$ Without the Brouwer-Kripke axiom of course.
} 
subcover.

PROOF: Let $(X, d)$ be a compact space, let $\mathcal{U}$ be a collection of open sets such that for every $x$ in $X$ belongs to a $U$ in $\mathcal{U}$. Since $(X, d)$ is compact, there is a uniformly continuous surjection $f$ from $\sigma_{2}$ to $X$. Then the collection:

$\left\{f^{-1}(U) \mid U \in \mathcal{U}\right\}$

is an open cover of $\sigma_{2}$. Therefore the following subset $B$ of $\overline{\bar{\sigma}}_{2}$ is a bar on $\sigma_{2}$ :

$B=\left\{\bar{\alpha}(n) \mid \exists U \in \mathcal{U} \forall \beta \in \sigma_{2}\left[\bar{\beta}(n)=\bar{\alpha}(n) \rightarrow \beta \in f^{-1}(U)\right]\right\}$

By BDD we know that $B$ descends from a decidable bar $C$, which by $\mathbf{F T}$ contains a finite bar. But then $B$ also contains a finite bar, namely the descent of the finite bar contained in $C$. Therefore $\mathcal{U}$ contains a finite subcover

COROLLARY: From BDD and FT it follows that every continuous function is continuous ${ }^{\mathrm{BRI}}$ and therefore continuous $^{\text {BIS }}$, or in other words: the three definitions coincide. So added to FT, the introduction of BDD finally resolves the continuity problem which sparked this article. Proof of the other statements in this subsection is given in the appendix, section 10 .

8.9 In [Veldman1985] a very readable description of Brouwer's justification of $\mathbf{B I}_{\mathbf{D}}$ is given. In fact, Brouwer more or less postulates an even more general axiom, from which one can derive $\mathbf{B I}_{\mathbf{D}}$. Veldman calls (part of) this even more general axiom 'Brouwer's Thesis'. We will present it below using our own terminology, and adopt the abbreviation BT (BT is classically true also, in fact it is equivalent to the combination of $\mathbf{B I}_{\mathbf{D}}$ and $\mathbf{B D D}$ ). For the presentation we need to introduce so-called genetic bars. (We will show later that the concept of a genetic bar coincides with the concept of a decidable thin bar). As the name suggests, the definition is a genetic one, and runs thus:

DEFINITION: A genetic bar is a bar obtained by application of the following two formation rules:

I. The trivial bar $\{\varangle \gg\}$ is a genetic bar.

II. If $B_{0}, B_{1}, \ldots$ is a sequence of genetic bars, then so is the bar $B$ given by: $B=\left\{n \star a \mid a \in B_{n}\right\}$.

These rules supply all the genetic bars.

The definition of genetic bars justifies the principle of genetic induction PGI, which runs as follows:

PGI The definition of genetic bars is valid. Moreover, if $\mathrm{P}$ is a property of bars, such that:

I. The trivial bar $\{\ll \triangleright\}$ has the property $\mathrm{P}$;

II. If $B_{0}, B_{1}, \ldots$ is a sequence of genetic bars with the property $\mathrm{P}$, then so is the bar $B$ given by: $B=\left\{n \star a \mid a \in B_{n}\right\}$;

then all genetic bars have the property $\mathrm{P}$.

Brouwer's Thesis can now be formulated like this:

BT Every bar descends from a genetic bar; and PGI holds. 
An intuitionistic plea for BT can be give in the following way. The intuitionistic universe $\sigma_{\omega}$ is inhabited by choice sequences arising step by step in the course of time. This means that in general only the minimum of information about an element is known. For the author, the axiom expresses that - given such a universe - the only one way to convince ourselves that a subset $B$ of $\overline{\bar{\sigma}}_{\omega}$ is indeed a bar, is to show that it descends from something that we can intuitively grasp as a bar, namely a genetic bar. ${ }^{31}$

Kleene calls this aptly 'reversing the arrows'. The genetic definition in fact mirrors what our intuition tries to do when visualising an arbitrary bar. The author has no trouble accepting this definition, and in this acceptance lies the immediate intuitive justification of PGI. Brouwer's justification looks rather more complex even when explained by Veldman, but we believe it to be essentially the same as our presentation above. Some nice examples (due to Kleene) of bars descending fom genetic bars are given in [Kleene\&Vesley1965], and more readable in [Veldman1985]. Kleene uses the terminology 'reversing the arrows' in connection with $\mathbf{B I}_{\mathbf{D}}$. We agree with Veldman, that the justification of $\mathbf{B T}$ is more intuitive than the justification of $\mathbf{B I}_{\mathbf{D}}$ given by Kleene, especially since the necessity of the decidability condition in $\mathbf{B I}_{\mathbf{D}}$ is left undiscussed in [Kleene\&Vesley1965]. Above we hope to have simplified the treatment in [Veldman1985].

Notice that BT is false in recursive mathematics. In the recursive universe, where far more information about each element is available than in the constructive and intuitionistic universe, it is possible to construct bars which do not descend from a genetic bar, see section 7 (these 'sparse recursive bars' capture every recursive element of $\sigma_{\omega}$, but do not form a bar in intuitionistic or classical mathematics; whereas any recursive genetic bar in recursive mathematics is also a bar in the constructive, classical and intuitionistic universe). This explains the failure of $\mathbf{B T}$ and therefore $\mathbf{B I}_{\mathbf{D}}$ in recursive mathematics (see the next theorem). In classical mathematics BT simply holds.

THEOREM: BT is equivalent to the combination of $\mathbf{B I}_{\mathbf{D}}$ and $\mathbf{B D D}$.

PROOF: First we show that BT implies BDD. For this we prove slightly more, using PGI, namely that every genetic bar is a decidable thin bar. The proof is easy. Clearly the trivial bar is a decidable thin bar. Now let $B_{0}, B_{1}, \ldots$ be a sequence of genetic bars which are decidable and thin. We must show that the genetic bar $B=\left\{n \star a \mid a \in B_{n}\right\}$ is likewise decidable and thin. Obviously the empty sequence $\varangle \bowtie$ is not in $B$. So let $a$ be in $\bar{\sigma}_{\omega}$, with length greater than zero. Then there is a $b$ in $\bar{\sigma}_{\omega}$ and an $n \in \mathbb{N}$ with $a=n \star b$. Now $a$ is in $B$ iff $b$ is in $B_{n}$. Therefore $B$ is decidable. To show that $B$ is thin, suppose that $a$ as above is in $B$, and that $c$ is in $\overline{\bar{\sigma}}_{\omega}$ with $c \sqsubset a$. We must show that $c$ is not in $B$. For $c$ equal to the empty sequence clearly $c$ is not in $B$. Else there is a $d$ in $\overline{\bar{\sigma}}_{\omega}$ with $c=n \star d$, and trivially $d \sqsubset b$ (remember $a=n \star b)$. Since $B_{n}$ is thin we find that $d$ is not in $B_{n}$, therefore $c$ is not in $B$. This shows that $B$ is thin. By PGI we conclude that every genetic bar is a decidable thin bar. And so BT implies that every bar descends from a decidable thin bar, which implies BDD.

Then we must show that $\mathbf{B T}$ implies $\mathbf{B I}_{\mathbf{D}}$. For this we turn to PGI, with a suitable property P. Let's say that a bar $C$ is inductable iff for every inductive $A$ such that $C \subseteq A$, we have that the empty sequence $\varangle \triangleright$ is in $A$. Clearly $\mathbf{B I}_{\mathbf{D}}$ is equivalent to the statement 'every decidable bar on $\sigma_{\omega}$ is inductable'. By BT this is equivalent to the statement 'every decidable bar on $\sigma_{\omega}$ which descends from

\footnotetext{
${ }^{31}$ In fact the more precise analysis is that there are two basic methods that can be employed in ascertaining the 'bar' status of a given subset $B$ of $\bar{\sigma}_{\omega}$. The direct method is to have $B$ given as a genetic bar. The other method is to see that $B$ descends from a previously ascertained bar. But these two methods put together yield the method of checking whether $B$ descends from a genetic bar.
} 
a genetic bar is inductable'. This last statement we will prove using PGI. Let's say that a genetic bar $B$ is inducing iff every decidable bar which descends from $B$ is inductable.

We now prepare the two basic ingredients of PGI. Firstly, clearly the trivial bar $\{\varangle \gg\}$ itself is inducing. Secondly let $B_{0}, B_{1}, \ldots$ be a sequence of genetic inducing bars. To apply PGI we must show that then the bar $B$ given by: $B=\left\{n \star b \mid b \in B_{n}\right\}$ is likewise inducing. This is not so hard. Let $C$ be a decidable bar descending from $B$. Let $A$ be an inductive subset of $\overline{\bar{\sigma}}_{\omega}$ such that $C \subseteq A$. Our task is to show that the empty sequence $\varangle \triangleright$ is in $A$. If $\varangle \gg \in C$ then we are done. Else put $C_{n}=\{c \mid n \star c \in C\}$, then $C_{n}$ is a decidable bar on $\sigma_{\omega}$ for each $n \in \mathbb{N}$. For each $n \in \mathbb{N}$ we have that $A_{n}=\{a \mid n \star a \in A\}$ is an inductive subset of $\overline{\bar{\sigma}}_{\omega}$. Since $C \subseteq A$, we find that $C_{n} \subseteq A_{n}$ and $C_{n}$ descends from $B_{n}$ for all $n \in \mathbb{N}$. By induction assumption this implies that $\varangle \gg$ is in $A_{n}$ for each $n \in \mathbb{N}$. Consequently $n$ is in $A$ for each $n \in \mathbb{N}$, and since $A$ is inductive we find that $\varangle \otimes$ is in $A$. Therefore $B$ is also inducing. We apply PGI to conclude that every genetic bar is inducing, and therefore by $\mathbf{B T}$ we have $\mathbf{B I}_{\mathbf{D}}$.

The other way around is more work. Assume $\mathbf{B D D}$ and $\mathbf{B I}_{\mathbf{D}}$. Now we must derive $\mathbf{B T}$. For this we first prove that every decidable thin bar is in fact a genetic bar. Therefore the notion 'decidable thin bar' and 'genetic bar' will be seen to coincide, making the definition of genetic bars valid (the first statement in PGI). So let $B$ be a decidable thin bar on $\sigma_{\omega}$. For each $a$ in $\overline{\bar{\sigma}}_{\omega}$, put $B_{a}=\left\{b \in \bar{\sigma}_{\omega} \mid a \star b \in B\right\}$. Now consider the set $A=\left\{a \in \bar{\sigma}_{\omega} \mid B_{a}\right.$ is a genetic bar $\}$. We hold that $A$ is an inductive set, which can be seen as follows. Suppose $a$ is in $\overline{\bar{\sigma}}_{\omega}$ such that $a \star n$ is in $A$ for each $n \in \mathbb{N}$. By the definition of $A$ this implies that $B_{a \star n}$ is a genetic bar for each $n \in \mathbb{N}$. But then $B_{a}$ is a genetic bar also, since $B_{a}=\left\{n \star b \mid n \in \mathbb{N}, b \in B_{a \star n}\right\}$. Therefore $a$ is in $A$, showing that $A$ is inductive. It is easily seen that $B$ is a subset of $A$, since $B_{b}$ is the trivial bar $\{\varangle \bowtie\}$ (and therefore genetic) for every $b$ in $B$, because $B$ is thin. So to round it up, we use $\mathbf{B I}_{\mathbf{D}}$ to conclude that the empty sequence $\varangle \triangleright$ is in $A$. By definition of $A$ this implies that $B$ is genetic. In combination with BDD and corollary 8.8 we now have that every bar descends from a genetic bar, which is the first half of BT.

For the second half of $\mathbf{B T}$ we must derive PGI from $\mathbf{B D D}$ and $\mathbf{B I}_{\mathbf{D}}$. The first step concerns the validity of the definition of genetic bars, but this has been dealt with above. Next, suppose $\mathrm{P}$ is a property of bars such that the trivial bar has property P. Suppose moreover that if $B_{0}, B_{1}, \ldots$ is a sequence of genetic bars having property $\mathrm{P}$, then the bar $B$ given by: $B=\left\{n \star a \mid a \in B_{n}\right\}$ likewise has property P. We must now show, using $\mathbf{B D D}$ and $\mathbf{B I}_{\mathbf{D}}$, that every genetic bar has the property $\mathrm{P}$. For this we refine our reasoning above in proving the first half of BT. Let $B$ be an arbitrary genetic bar. Then by BDD (and corollary 8.8) $B$ descends from a decidable thin bar $B^{\prime}$. Consider the set $A=\left\{a \in \bar{\sigma}_{\omega} \mid\right.$ every genetic bar which descends from $B_{a}^{\prime}$ has property $\left.\mathrm{P}\right\}$. We hold that $A$ is an inductive set, which can be seen as follows. Suppose $a$ is in $\overline{\bar{\sigma}}_{\omega}$ such that $a \star n$ is in $A$ for each $n \in \mathbb{N}$. By the definition of $A$ this implies that for each $n \in \mathbb{N}$, every genetic bar descending from $B_{a \star n}^{\prime}$ has property $\mathrm{P}$.

Now let $C$ be an arbitrary genetic bar descending from $B_{a}^{\prime}$. To show that $a$ is in $A$ we must show that $C$ has property $\mathrm{P}$. We distinguish two cases, following the definition of genetic bars. The first case is that $C$ is the trivial bar. Then trivially $C$ has property $\mathrm{P}$. The second case is that there is a sequence of genetic bars $C_{0}, C_{1}, \ldots$ such that $C=\left\{n \star c \mid n \in \mathbb{N}, c \in C_{n}\right\}$. Now it is easy to see that $C_{n}$ descends from $B_{a \star n}^{\prime}$ for each $n \in \mathbb{N}$, and therefore by the assumption on $a$ has property $\mathrm{P}$. Then $C$ has property $\mathrm{P}$ also, by the assumption on $\mathrm{P}$. Since $C$ was arbitrary, this shows that $a$ is in $A$, showing that $A$ is inductive. We hold that $B^{\prime}$ is a subset of $A$. To see this, let $b$ be in $B^{\prime}$. Since $B_{b}^{\prime}$ is the trivial bar $\{\varangle \gg\}$ (remember $B^{\prime}$ is thin), every genetic bar descending from $B_{b}^{\prime}$ has to be the trivial bar also, and so has property $\mathbf{P}$. So to sum it up, we use $\mathbf{B I}_{\mathbf{D}}$ to conclude that the empty 
sequence $\varangle \diamond$ is in $A$. By definition of $A$ this implies that every genetic bar descending from $B^{\prime}$ has property $\mathrm{P}$, and so $B$ has property P. This proves PGI, the second half of BT, and so we have derived BT from $\mathbf{B D D}$ and $\mathbf{B I}_{\mathbf{D}}$ as promised

8.10 With the focus on a reunion of the antipodes, we reask the question posed in 2.4: why not accept Markov's Principle in intuitionism as well? The author thinks this will be a fruitful change to intuitionism $^{32}$. We present a first consequence of $\mathbf{M P}$, which is in fact an equivalence.

PROPOSITION: The following statements are equivalent:

(i) Markov's Principle MP.

(ii) 'Every function $f$ from a metric space $X$ to another metric space $Y$ is strongly extensional, that is: for all $x, y$ in $X$ : if $f(x) \# f(y)$ then $x \# y$ '.

We think one should have started by taking the strong extensionality in (ii) as the definition of a function, and this only illustrates the force of intuition behind MP; just as theorem 1.3 illustrates the force of intuition behind FT. Notice that for continuous functions strong extensionality is a triviality. We advocate that strong extensionality become the standard definition for constructive functions, even if one doesn't accept MP. Reserving the term 'weak function' for any function which is only known to 'send equivalents to equivalents', we believe we will never come across a weak function for which we cannot conclude it is a (strongly extensional) function ${ }^{33}$. In this way static in constructivism is reduced.

8.11 Banach's 'open mapping theorem', which has received a lot of constructive attention, is an interesting case for the antipodes. It holds, mutatis mutandis, in CLASS, INT and RUSS. In [Ishihara1994] a nice weaker version is proved in BISH, this version concerns what we will call the sequential topology (see subsection 8.17), rather than the metric topology. In CLASS and INT, one usually proves the theorem using a contrapositive form of Baire's category theorem ${ }^{34}$. Classically this form of Baire's category theorem is derived from PEM, intuitionistically it follows from CP. It is a powerful topological insight, which certainly deserves a place in a reunion of the two antipodes. ${ }^{35}$ We present it below as an axiom derived from $\mathbf{C P}$.

CP Baire $_{\text {Let }}\left(A_{n}\right)_{n \in \mathbb{N}}$ be an enumerable collection of closed subsets of a complete metric space $X$, such that each $x$ in $X$ is in some $A_{n}$. Then there is an $A_{n}$ which contains an open ball in the metric given.

REMARK: The attentive reader will see our strategy. Historically INT and CLASs have been presented as incompatible, and intuitionism has been called 'the revolution'. Intuitionism has also been called 'mystic'. But when we analyze, we find that almost all intuitionistic axioms are valid classically as well. It is 'only' $\mathbf{C P}$ which is essentially responsible for intuitionistic results which do not hold in CLASS. And with $\mathbf{C P}_{\text {Baire }}$ we isolate from $\mathbf{C P}$ yet another powerful topological tool which is valid in CLASS, thus

\footnotetext{
${ }^{32}$ It is true that Markov's Principle conflicts with the Brouwer-Kripke axiom, but it so gives just another motivation for the rejection of this Brouwer-Kripke axiom (the full $\mathrm{BK}$ axiom also conflicts with $\mathbf{A C} \mathbf{C}_{11}$ ).

${ }^{33}$ The formalization of this belief is exactly MP, by the above proposition.

${ }^{34}$ Ishihara's version gives an interesting though slightly weaker alternative.

${ }^{35}$ The contrapositive form is false in RUss, even though Baire's category theorem holds in BISH. We have not found a 'strong enough in our eyes' adaptation which holds in all three antipodes. The reader may consult [Bridges\&Richman1987] for various adaptations which do hold in all three antipodes.
} 
further reducing the distance of INT to CLASS. The distance the other way round is however still quite large, due to the use in CLASS of PEM outside of decidable situations.

PROposition: $\mathbf{C P}_{\text {Baire }}$ fails in Russ.

PROOF: Consider for $n \in \mathbb{N}$ the closed subset $A_{n}=\left\{\alpha \in \sigma_{\omega} \mid \forall m \in \mathbb{N}\left[\alpha(m)=\phi_{n}(m)\right]\right\}$ of $\sigma_{\omega \mathrm{REC}}$

8.12 One other matter needs to be settled to finish our proposal for reuniting (some of) the antipodes, as far as this simple paper goes. This concerns axioms of choice. In BISH (cf. [Bishop1967]) some very liberal axioms of choice are formulated, which place little to no restriction on the 'domain' and 'range' of the situation ${ }^{36}$. Contrary to the popular claim that BISH is a common theory for intuitionism, classical and recursive mathematics, we will conclude below that these liberal axioms of choice are unacceptable from both a recursive and an intuitionistic point of view. Let us put forward Bas Spitters' argument why even a weak, non-continuous axiom of choice will fail in recursive mathematics (many authors have commented on similar situations).

PROPOSITIOn: (Bas Spitters) There is a subset $A$ of $\sigma_{\omega \mathrm{REC}} \times \mathbb{N}$, such that $\forall \alpha \in \sigma_{\omega \mathrm{REC}} \exists n \in N[(\alpha, n) \in A]$ and yet there is no recursive function $f$ from $\sigma_{\omega \mathrm{REC}}$ to $\mathbb{N}$ which for all $\alpha$ in $\sigma_{\omega \mathrm{REC}}$ gives us that $(\alpha, f(\alpha)) \in A$.

PROOF: Take $A=\left\{(\alpha, e) \in \sigma_{\omega \mathrm{REC}} \times I(\mathbb{N}, \mathbb{N}) \mid e\right.$ is a recursive index of $\left.\alpha\right\}$. Clearly $\forall \alpha \in \sigma_{\omega \mathrm{REC}} \exists n \in N[(\alpha, n) \in A]$. But if there were a choice function $f$ from $\sigma_{\omega \mathrm{REC}}$ to $\mathbb{N}$ which for all $\alpha$ in $\sigma_{\omega \mathrm{REC}}$ gives us that $(\alpha, f(\alpha)) \in A$, then for instance equivalence of two elements $\alpha, \beta$ of $\sigma_{\omega \mathrm{REC}}$ (meaning that $\alpha(n)=\beta(n)$ for each $n \in \mathbb{N}$ ) would be decidable, since one would only have to check whether $f(\alpha)$ equals $f(\beta)$ or not. This decidability of equivalence contradicts well-known basic results in recursive-function theory •

This proposition goes to show that one cannot put forward any 'conventional' axiom of choice to hold in BISH. Before we meditate on possible solutions, we first investigate the intuitionistic situation. In intuitionism, the intuition behind $\mathbf{A C}_{11}$ (and $\mathbf{A C}_{10}$ ) depends vitally on the fact that the assertion $\exists y[A(x, y)]$ holds for all $x$ in all of $\sigma_{\omega}$, that $\sigma_{\omega}$ is a non-deterministic universe with incomplete information, and that $A$ is a subset of $\sigma_{\omega} \times \sigma_{\omega}$. An intuitionist is definitely not convinced of the validity of choice axioms for 'arbitrary domains' and 'arbitrary ranges', and this is vindicated by the proposition above (notice that $\sigma_{\omega \mathrm{REC}}$ is a subset of $\sigma_{\omega}$ ). The axiom $\mathbf{D} \mathbf{C}_{1}$ is therefore also phrased in the context of elements and subsets of $\sigma_{\omega}{ }^{37}$. $\mathbf{D C}_{1}$ is weaker than the axiom of dependent choice currently accepted in BISH, so we feel that $\mathbf{D} \mathbf{C}_{1}$ can be safely adopted for any reunion of antipodes.

8.13 A way out of some of the problems above can be found in the presentation axiom described in [Troelstra\&vanDalen1988] (sect. 4.2). If we rephrase and restrict a little, then basically this axiom states the following (where the notion of 'set' is left vague):

\footnotetext{
${ }^{36}$ Many authors have commented on this, but there has been no formal change of phrasing of these axioms. In practice only the weaker forms that we propose further on seem to be really used.

${ }^{37}$ For 'higher-order' settings even the combination of $\mathbf{A} \mathbf{C}_{11}$ and $\mathbf{D} \mathbf{C}_{1}$ does not always suffice, even if these settings seem intuitively clear. It probably is worthwile to investigate if intuition allows for higher-order choice axioms than $\mathbf{A C}_{11}$ and $\mathrm{DC}_{1}$
} 
PA Let $A$ be a set. Then we can find a subset $C$ of $\sigma_{\omega}$, and an equivalence relation $\equiv_{A}$ on $C$, such that

(1) $A$ coincides with $C$ equipped with $\equiv_{A}$, under a mapping $h$ from $C$ to $A$,

(2) If $B$ is a subset of $A \times \sigma_{\omega}$ such that $\forall \alpha \in A \exists \beta \in \sigma_{\omega}[(\alpha, \beta) \in B]$, then there is a function $f$ from $C$ equipped with $\equiv_{\omega}$ to $\sigma_{\omega}$ such that $\forall \alpha \in A \exists \gamma \in C[h(\gamma)=\alpha \wedge(\alpha, f(\gamma)) \in B]$.

Informally speaking, the presentation axiom states that for every set $A$, there is an underlying 'presentation' set $C$ for which an axiom of choice holds, even if it fails for $A$. By $C$ being an 'underlying presentation' we mean that $C$ is a subset of $\sigma_{\omega}$ such that $A$ can be derived from $C$ by imposing an equivalence relation $\equiv_{A}$ on $C$. The reader may check that (only) for the example in the proof of proposition 8.12, taking TOT as presentation of $\sigma_{\omega \mathrm{REC}}$ would resolve the problem (consider $\mathbb{N}$ as a subset of $\sigma_{\omega}$, then TOT is also a subset of $\sigma_{\omega}$ and take $\left.s \equiv{ }_{A} t=\forall n \in \mathbb{N}\left[\phi_{s}(n)=\phi_{t}(n)\right]\right)$. But we have little faith in the usefulness of the presentation axiom, since it does not offer enough to resolve the choice problem for RUss. This because in RUss choice functions must be partial recursive functions, otherwise they are of little use. And if one exacts this in the conclusion of PA, then PA is false in RUss (see [Troelstra\&vanDalen1988] (sect. 4.11)).

Another way to solve many problems is by taking the desired result of a possible choice axiom (that there is a partial recursive choice function) as a premise in statements one wants to prove. For example, instead of saying 'every open cover of a complete metric space has an enumerable subcover' one can also state: 'every effective open cover of a complete metric space has an enumerable subcover', where 'effective' means 'given by a suitable algorithm'. So once again, the discussion is whether to adopt axioms, or to restrict one's attention beforehand to objects already having the desired properties. In the case of choice axioms for Russ, the latter seems an elegant enough way. Nonetheless we formulate an intuitively motivated axiom of choice for RUSs, called $\mathbf{C T}_{11}$. With this axiom one can prove BDD (see the appendix, $\mathbf{C T}_{11}$ is not needed for the very similar statement 'every effective bar descends from a decidable bar'). We now go into this matter in greater detail.

The different nature of recursive mathematics is revealed by the fact that in RUSS there is indeed a nontrivial underlying domain for $\sigma_{\omega}$, namely the subset TOT of $\mathbb{N}$ of indices of total recursive functions. This is not the case in classical and intuitionistic mathematics. The set TOT however is a truly difficult mathematical entity from a constructive point of view. It is not even an enumerable subset of $\mathbb{N}$, let alone decidable. So it cannot be constructed on its own. In order to work with total recursive functions one has to consider the partial recursive functions as well. Now the choice problem comes to the fore, but it can be resolved with the axiom $\mathbf{E C T}_{0}$ (see 2.4) which is broadly accepted in RUss. From $\mathbf{E C T}_{0}$ it follows that if for all $n$ in TOT a certain natural number exists, then there is already a partial recursive function from $\mathbb{N}$ to $\mathbb{N}$ which for $n$ in TOT yields precisely such a number. However, the axiom $\mathbf{E C T}_{0}$ is motivated in [Troelstra\&vanDalen1988] (sect. 4.4) only in the formal logical setting of being consistent with Heyting Arithmetic (HA). The author finds the phrasing and the motivation unappealing. We believe that an easier and more intuitive motivation can be given for a straightforward choice axiom. Hence we phrase an 'axiom of choice for the underlying domain $\mathbb{N}^{\prime}$ for Russ.

$\mathbf{C T}_{11}$ Let $A$ be a subset of $\mathbb{N} \times \mathbb{N}$ such that

$\forall n \in$ TOT $\exists m \in \mathbb{N}[(n, m) \in A]$.

Then there is a partial recursive function $h$ from $\mathbb{N}$ to $\mathbb{N}$ such that for all $n \in$ TOT we have: $(n, h(n)) \in A$. 
We motivate this axiom as follows. Given $\forall n \in \mathbf{T O T} \exists m \in \mathbb{N}[(n, m) \in A]$, we analyze the way in which the information $n \in$ TOT can lead to the pinpointing of an $m \in \mathbb{N}$ for which $(n, m) \in A$. Since such an $m$ must be produced at a finite point in time, we cannot have used more information than $n$ itself and a finite number $k$ of computations of $\phi_{n}$ say on the input numbers $0,1 \ldots, M$. So the method which produces the desired $m \in \mathbb{N}$ does not depend on the fact that $n$ is in TOT, but on a finite number of succesful computations derived from $n^{38}$.

If we stipulate a canonical way to compute (e.g. we first take one computational step in computing $\phi_{n}(0)$, then we compute the first step in $\phi_{n}(1)$, then another step in $\phi_{n}(0)$, then another in $\phi_{n}(1)$ and a step in $\phi_{n}(2)$, then back to $\phi_{n}(0)$ etc.), then we arrive at the following conclusion. Given $n$ and a finite number $k$ of computations derived from $n$ we know whether this finite number $k$ is sufficient to produce $m \in \mathbb{N}$ or not. Our method either says: 'ok, enough information' or it says 'too bad, need some more information'. Therefore there is a decidable subset $B$ of $\mathbb{N} \times \mathbb{N}$ such that for all $(n, k) \in B$ we have: the number $k$ of computations derived from $n$ is sufficient to produce an $m \in \mathbb{N}$ such that $n \in$ TOT implies $(n, m) \in A$.

Now put $A^{\prime}=\{(n, m) \in \mathbb{N} \mid n \in \mathbf{T O T} \rightarrow(n, m) \in A\}$. By our reasoning above we find:

$\forall n \in \mathbb{N}\left[\exists k \in \mathbb{N}[(n, k) \in B] \rightarrow \exists m \in \mathbb{N}\left[(n, m) \in A^{\prime}\right]\right]$

Then we can apply $\mathbf{C T}_{01}$, which we formally derived from $\mathbf{C T}_{0}$ in paragraph 2.4, to produce a partial recursive $h$ from $\mathbb{N}$ to $\mathbb{N}$ such that for all $n \in \mathbb{N}$ : if $\exists k \in \mathbb{N}[(n, k) \in B]$ then $h(n)$ is defined and $(n, h(n)) \in A^{\prime}$. Notice that $h$ then is as required in the conclusion of $\mathbf{C T}_{11}$, by the definition of $A^{\prime}$.

REMARK: In our reasoning we have reduced $\mathbf{C T}_{11}$ to the core of RUSS, namely $\mathbf{C T}$. Notice that $\mathbf{C} \mathbf{T}_{11}$ is not an instance of the presentation axiom. We do not see a way to phrase a common axiom of choice for all three antipodes which for RUss amounts to $\mathbf{C T}_{11}$. That our terminology is more or less consistent is the content of the following proposition.

PRoposition: $\mathbf{C T}_{0}$ follows from $\mathbf{C T}_{11}$.

PRoOF: Let $A$ be a subset of $\mathbb{N} \times \mathbb{N}$ such that $\forall n \in \mathbb{N} \exists m \in \mathbb{N}[(n, m) \in A]$. Then in particular we find: $\forall n \in$ TOT $\exists m \in \mathbb{N}\left[\left(\phi_{n}(0), m\right) \in A\right]$. Put $A^{\prime}=\left\{(n, m) \in \mathbf{T O T} \times \mathbb{N} \mid\left(\phi_{n}(0), m\right) \in A\right\}$, then trivially:

$\forall n \in$ TOT $\exists m \in \mathbb{N}\left[(n, m) \in A^{\prime}\right]$

So by $\mathbf{C T}_{11}$ there is a recursive function $f$ from $\mathbb{N}$ to $\mathbb{N}$ such that for each $n \in \mathbf{T O T}:(n, f(n))$ is in $A^{\prime}$. It is standard to construct a recursive function $g$ from $\mathbb{N}$ to $\mathbb{N}$ such that for each $n \in \mathbb{N}$ we have: $\forall m \in \mathbb{N}\left[\phi_{g(n)}(m)=n\right]$, and therefore $g(n) \in \mathbf{T O T}$. Now the function $h$ given by $h=f \circ g$ is a recursive function from $\mathbb{N}$ to $\mathbb{N}$ such that for all $n \in \mathbb{N}$ we have: $(n, h(n)) \in A$

8.14 Next we turn to CLASS and INT. Since their basis is more conventional than RUSS, there is a conventional way to phrase an axiom of general choice for the reunion of classical and intuitionistic mathematics. Such an axiom of general choice must be restricted in a way comparable to $\mathbf{A C}_{11}$ : the 'domain' of choice should be limited to a spread ( $\sigma_{\omega}$ is sufficient). However the intuitionistic insight that the resulting choice function is continuous, must of course be dropped for the reunion. So we first

\footnotetext{
${ }^{38}$ But the conclusion that $(n, m) \in A$ can depend on the fact that $n$ is in TOT! For instance if $A$ is specified as a subset of $\mathbf{T O T} \times \mathbb{N} \ldots$
} 
propose a weak version of $\mathbf{A C}_{11}$, which in the conclusion drops the continuity of the choice function (so it leaves open whether the choice function is a spread-function).

Let $A$ be a subset of $\sigma_{\omega} \times \sigma_{\omega}$ such that:

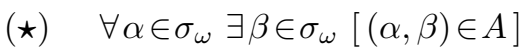

Then there is a function $\gamma$ from $\sigma_{\omega}$ to $\sigma_{\omega}$ such that for each $\alpha$ in $\sigma_{\omega}:(\alpha, \gamma(\alpha))$ is in $A$. We say that $\gamma$ fulfills $(\star)$.

REMARK: Because it is not asserted that the choice function is a spread-function, the choice function cannot be seen to be representable by an element of $\sigma_{\omega}$. We have not investigated the consequences of this, which might be further-reaching than expected at first glance (but see some propositions below). We have that $\mathbf{A} \mathbf{C}_{11}$ is equivalent to the combination of $\mathbf{A} \mathbf{C}_{11 \text { weak }}, \mathbf{A C}_{10}$ and $\mathbf{A C}_{01} . \mathbf{A} \mathbf{C}_{10}$ in turn is equivalent to the combination of $\mathbf{C P}, \mathbf{B D D}$ and $\mathbf{A} \mathbf{C}_{00}$.

Using BDD we can prove some interesting propositions, which for all three antipodes can serve as alternative 'axioms' of continuous choice. The first proposition is a surrogate for $\mathbf{A C}_{10}$. Notice that its proof is also a proof that $\mathbf{A C}_{10}$ follows from the combination of $\mathbf{C P}, \mathbf{B D D}$ and $\mathbf{A} \mathbf{C}_{00}$.

PRoposition: (using BDD) Let $A$ be a subset of $\sigma_{\omega} \times \mathbb{N}$ such that:

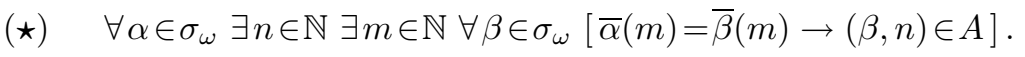

Then there is a spread-function $\gamma$ from $\sigma_{\omega}$ to $\mathbb{N}$ such that for each $\alpha$ in $\sigma_{\omega}:(\alpha, \gamma(\alpha))$ is in $A$.

PRoOF: From ( $\star$ ) we deduce that the set $B=\left\{a \in \overline{\bar{\sigma}}_{\omega} \mid \exists n \in \mathbb{N} \forall \beta \in \sigma_{\omega} \cap a[(\beta, n) \in A]\right\}$ is a bar on $\sigma_{\omega}$. By lemma 8.8 the bar $B$ descends from a decidable thin bar $C$. By definition of $B$, for each $c$ in $C$ there is an $n \in \mathbb{N}$ such that $\left.\forall \beta \in \sigma_{\omega} \cap c[(\beta, n) \in A]\right\}$. Now by $\mathbf{A C}_{00}$ the desired spread-function $\gamma$ can easily be defined on $C$

COROLLARY: Every continuous function from a spreadlike metric space to another metric space can be represented by a spread-function.

8.15 The second proposition, which for all three antipodes can serve as a surrogate for $\mathbf{A C}_{11}$, closely resembles the Michael Selection Theorem. In [Waaldijk1996] an intuitionistic version of the Michael Selection Theorem is proved, using $\mathbf{A C}_{10}$ and $\mathbf{D} \mathbf{C}_{1}$ (but by corollary 8.14 one sees that in fact only BDD is used, so the theorem holds in all three antipodes). This theorem is concerned with conditions under which a continuous choice function may be constructed, given a subset $A$ from a product space $X \times Y$ such that for all $x$ in $X$ there is a $y$ in $Y$ with $(x, y) \in A$. The Michael theorem provides a continuous choice function ${ }^{39}$ under the following conditions. Firstly $X$ must be a spreadlike metric space and $Y$ must be a Banach space. Moreover the set $A$ is 'Lower Semi Continuous', and for each $x$ in $X$ the set $\{y \in Y \mid(x, y) \in A\}$ is convex and closed. The proposition below indicates that for $X=\sigma_{\omega}=Y$, lower semicontinuity and the closedness condition suffice for the existence of a suitable continuous choice function. To make this precise, we use a simplified definition of lower semicontinuity ${ }^{40}$.

\footnotetext{
${ }^{39}$ Such a function is called a continuous selection.

${ }^{40}$ For the general metric definition, see [Waaldijk1996].
} 
Definition: Let $A$ be a subset of $\sigma_{\omega} \times \sigma_{\omega}$ such that $\forall \alpha \in \sigma_{\omega} \exists \beta \in \sigma_{\omega}[(\alpha, \beta) \in A]$. Then $A$ is called lower semicontinuous iff for all $(\alpha, \beta) \in A$ and for all $n \in \mathbb{N}$, there is an $m \in \mathbb{N}$ such that: $\forall \gamma \in \sigma_{\omega}[\bar{\gamma}(m)=\alpha(m) \rightarrow$ $\left.\exists \delta \in \sigma_{\omega}[\bar{\delta}(n)=\bar{\beta}(n) \wedge(\gamma, \delta) \in A]\right]$.

Proposition: (Michael Selection Theorem for $\sigma_{\omega}$, using BDD and $\mathbf{D C}_{1}$ ) Let $A$ be a lower semicontinuous subset of $\sigma_{\omega} \times \sigma_{\omega}$, such that for all $\alpha$ in $\sigma_{\omega}$ the set $\left\{\beta \in \sigma_{\omega} \mid(\alpha, \beta) \in A\right\}$ is closed. Then there is a continuous (spread-)function $\gamma$ from $\sigma_{\omega}$ to $\sigma_{\omega}$, such that for all $\alpha$ in $\sigma_{\omega}$ we have $(\alpha, \gamma(\alpha)) \in A$.

PROOF: The proof is a simplified transposition of the constructive proof of the Michael theorem in [Waaldijk1996]. By definition we have:

$\forall \alpha \in \sigma_{\omega} \forall n \in \mathbb{N} \exists m \in \mathbb{N} \exists \beta \in \sigma_{\omega} \forall \gamma \in \sigma_{\omega}\left[\bar{\gamma}(m)=\alpha(m) \rightarrow \exists \delta \in \sigma_{\omega}[\bar{\delta}(n)=\bar{\beta}(n) \wedge(\gamma, \delta) \in A]\right]$.

Therefore for $n \in \mathbb{N}$ the set $A_{n}=\left\{(\alpha, a) \mid \lg (a)=n \wedge \exists \beta \in \sigma_{\omega} \cap a[(\alpha, \beta) \in A]\right\}$ satisfies the requirements of proposition 8.14 above. So the set $R_{n}=\left\{\gamma \in \sigma_{\omega} \mid \gamma\right.$ is a spread-function $\wedge \forall \alpha \in \sigma_{\omega}\left[\left(\alpha, \gamma(\alpha) \in A_{n}\right]\right\}$ is inhabited for each $n \in \mathbb{N}$.

This shows that for each $n \in \mathbb{N}$ we can find a spread-function $\delta$ in $\sigma_{\omega}$ which for each $\alpha$ in $\sigma_{\omega}$ give the first $n$ values of a $\beta$ such that $(\alpha, \beta)$ is in $A$. Now the lower semicontinuity of $A$ guarantees us that given such a $\delta$, we can always 'extend' $\delta$ to a spread-function $\delta^{\prime}$ which for each $\alpha$ gives us the first $n+1$ values of a $\beta$ with $(\alpha, \beta)$ in $A$, and such that $\delta(\alpha) \sqsubset \delta^{\prime}(\alpha)$. Therefore we can apply $\mathbf{D C}_{1}$ to find a sequence of spread-functions $\left(\gamma_{n}\right)_{n \in \mathbb{N}}$ such that for all $\alpha$ in $\sigma_{\omega}$ and all $n \in \mathbb{N}$ we have both $\exists \beta \in \sigma_{\omega}\left[\bar{\beta}(n)=\gamma_{n}(\alpha) \wedge(\alpha, \beta) \in A\right]$ and $\gamma_{n}(\alpha) \sqsubset \gamma_{n+1}(\alpha)$. It is trivial to construct from $\left(\gamma_{n}\right)_{n \in \mathbb{N}}$ a spread-function $\gamma$ from $\sigma_{\omega}$ to $\sigma_{\omega}$ such that for all $n \in \mathbb{N}$ and $\alpha$ in $\sigma_{\omega}$ one has $\overline{\gamma(\alpha)}(n)=\gamma_{n}(\alpha)$. So then we finally apply the fact that for all $\alpha$ in $\sigma_{\omega}$ the set $\left\{\beta \in \sigma_{\omega} \mid(\alpha, \beta) \in A\right\}$ is closed to conclude that $\gamma$ is the required spread-function $\bullet$

REMARK: The proof of this proposition illustrates the necessity of surrogates for $\mathbf{A C}_{10}$. The axiom $\mathbf{D C}_{1}$ is strict in the sense that it requires the objects which one would chain together to be representable by elements of $\sigma_{\omega}$. For continuous functions from a spreadlike metric space to another metric space, this representability is the case, by corollary 8.14 .

8.16 All the axiomatic ingredients having been presented, we now ask attention for four axiomatic systems. First of all we shortly plead for $\mathrm{BISH}^{\star}$, which is $\mathrm{BISH}$ with $\mathbf{A C}_{01}$ and $\mathbf{D} \mathbf{C}_{1}$ as axioms of choice rather than the ones currently in use. The advantage of this axiomatic system is that practically everyone will agree to its axioms. The leaps of faith required are relatively small. The drawback is that intuition gets little space, therefore certain intuitive truths cannot be deduced. As stated, we recommend a different approach towards continuous functions for this system.

8.17 Next we shortly plead for a formal system which incorporates as much common intuition of the three antipodes ClASs, RUSS and INT as possible, with the addition of MP. Let us call this system $\mathrm{BISH}^{+}$. The axioms of $\mathrm{BISH}^{+}$are therefore: the axioms of $\mathrm{BISH}^{\star}, \mathbf{M P}$, and BDD. ${ }^{41}$ The advantage of $\mathrm{BISH}^{+}$is that results derived 'hold in reality' independently of whether reality is deterministic or nondeterministic (see section 7). Another advantage is that a common framework for all three antipodes can be developed in such a way that work done in one antipode becomes transferable to another antipode

\footnotetext{
${ }^{41}$ Intuitionists which do not believe in MP have the option to leave this out of course.
} 
to the extent possible. This system can be extended with 'common insights', to give an oversight of what theorems hold in all three antipodes.

One such 'common insight' of the three antipodes is that in a complete metric space the so-called sequential topology coincides with the metric topology ${ }^{42}$. Therefore every sequentially continuous function from a complete metric space to another metric space is a continuous function. Let us explain shortly what is meant by 'sequential topology', since in the literature this definition does not occur. A subset $A$ of a metric space $(X, d)$ is sequentially open iff for every $a$ in $A$, and every Cauchy-sequence $\left(a_{n}\right)_{n \in \mathbb{N}}$ in $(X, d)$ converging towards $a$, there is an $N \in \mathbb{N}$ such that for all $m \in \mathbb{N}, m>N$, we have $a_{m}$ is in $A$.

We do not see how to turn this common insight into (or prove it from) an axiom which has a simple defense. The same goes for Banach's open mapping theorem, which also holds in all three antipodes.

REMARK: In [Waaldijk1996] the so-called apartness topology is introduced as follows. Let $(X, \#)$ be an apartness space. Let $A$ be a subset of $X$. Then $A$ is open in $(X, \#)$ iff for all $x$ in $A$ we have: $\forall y \in X[y \in A \vee y \# x]$. It is easy to prove in BISH that every (strongly extensional) function from a topological apartness space to another topological space is continuous. Now in both INT and RUSS one can show that for a complete metric space the metric topology coincides with the apartness topology. From this one arrives at the conclusion that every function from a complete metric space to another topological space is continuous.

8.18 Of course we plead for a formal system which is a reunion of CLASS and INT with the addition of MP. ${ }^{43}$ Let us call this system CLINT ${ }^{+}$. Then the axioms of $\mathrm{CLINT}^{+}$are the axioms of $\mathrm{BISH}^{+}$, plus $\mathbf{A C}_{11 \text { weak }}, \mathbf{B T}$ and $\mathbf{C} \mathbf{P}_{\text {Baire }}$. This system develops a substantial part of classical mathematics in an intuitionistic way, such that both classical and intuitionistic mathematicians can benefit. Therefore this system comes closest to what we believe was Bishop's primary aim.

8.19 Finally, we plead for traditional intuitionism INT with the addition of MP. In the author's personal opinion INT is the branch of constructive mathematics which is closest to reality. In a world of incomplete information the axiom $\mathbf{C P}$ is an unmistakeable constructive truth, that merits serious study. The addition of MP is a simple consequence of the same attitude: if an axiom has a clear intuitive defense, then this axiom is worth adopting to study its consequences.

\section{Conclusions and ACKnowledgements}

9.1 To really open a discussion, we sum up what we feel to be the most important conclusions to be drawn from this paper. They are presented directly below as numbered statements, meant to draw attention.

\footnotetext{
${ }^{42}$ From this insight it follows that every surjective linear function with located kernel from a Banach space to another Banach space, is open. In other words: a 'located kernel' version of Banach's open mapping theorem, see [Ishihara1994].

${ }^{43}$ Intuitionists which do not believe in MP have the option to leave this out of course.
} 
1. The intuition that with regard to continuous functions one can restrict oneself to continuous functions which are uniformly continuous on compact (sub)spaces is in fact equivalent to the intuition behind the fan theorem FT (within BISH). This intuition has so far been the very cornerstone of BISH. Therefore BISH implicitly implies the fan theorem FT. It is not yet clear whether a 'satisfactory' theory of continuous functions on compact spaces can be built without explicitly relying on the fan theorem. To understand some of the problems see theorem 1.3. But also see section 7 , since 'satisfactory' obviously depends on our view of reality.

2. This foundational issue should be studied with priority. Several possibilities need investigation.

3. One important possibility is that of 'reuniting the antipodes'. Hereby we mean the adoption of a simple set of axioms which, when suitably interpreted, are acceptable both intuitionistically and classically. Sweeping aside historical misunderstandings, such a constructive mathematics can be much more powerful than BISH currently is, both mathematically and communicatively speaking.

4. For the purpose of such a reunion we propose the system $\mathrm{CLINT}^{+}$, with axioms $\mathbf{B T}, \mathbf{C P}_{\text {Baire }}, \mathbf{M P}$, $\mathbf{A C}_{11 \text { weak }}, \mathbf{D C}_{1}$, and Ind, at least for the time being.

5. CP is the only axiomatic insight of intuitionism which is not classically acceptable ${ }^{44}$. This simply means that $\mathbf{C P}$ is a truly original intuition. Adding $\mathbf{C P}$ to the above list we get full-blown INT with MP added. This combination, called $\mathrm{INT}^{+}$, is worth serious study with regard to all fields of mathematics.

6. The link between reality and foundations of mathematics can be studied experimentally (see section 7). On the other hand, the link between reality and foundations of physics can be studied mathematically. This can have a profound impact on physics and mathematics. Physicists therefore should be aware of foundational issues in mathematics, and mathematicians should be aware of foundational issues in physics.

9.2 I would like to thank the following people for their support in the epigenesis of this paper. Firstly Henk Taale, fellow mathematician at the Transport Research Centre, for convincing me to write this paper at all. Then of course Wim Veldman, who was my thesis supervisor and much more, for his continuing interest in this topic. In the last three years Bas Spitters joined the discussions, and like Wim gave many valuable comments. Their friendly help and criticism have greatly improved the quality of the presentation. Peter Schuster contributed his enthusiasm, which has been an important stimulant in the whole thing, and his apt terminology 'reuniting the antipodes' which enabled me to find a good focus for the discussion. I am happy that Wim Couwenberg also became interested, his enthusiastic support means a lot to me. Also, I thank the anonymous referee, who made kind and very helpful remarks, and thoughtfully included some extra references on history and on determinism.

Since 1993 my spiritual master Parthasarathi Rajagopalachari has helped me find a direction in mathematics which is close to my heart and my intuition. Of course this is only a byproduct of his help in more important aspects of life than mathematics.

I also wish to thank my friends Thijs and Reinier, who were more than casually interested both in the contents and the proceedings of this paper. Finally I wish to thank my children Nora and Femke and my wife Suzan for their patience with this neverending project, and for their love.

\footnotetext{
${ }^{44}$ We intend to show in a follow-up paper that there is a simple classical game-theoretic setting, in which all intuitionistic axioms hold true classically (including $\mathbf{C P}$ ).
} 


\section{APPENDIX: REMAINING PROOFS AND REMARKS}

10.1 We still owe the reader the proof of theorem 6.2. For the proof we will draw freely from the theory expounded in [Kleene1952]. There is a decidable subset $I(\mathbb{N},\{0,1\})$ of $\mathbb{N}$ such that each $e$ in $I(\mathbb{N},\{0,1\})$ is a properly formed recursive index of a partial recursive function from $\mathbb{N}$ to $\{0,1\}$, and vice versa for each partial recursive function $\alpha$ from $\mathbb{N}$ to $\{0,1\}$ there is an $e$ in $I(\mathbb{N},\{0,1\})$ such that $\forall n \in \mathbb{N}\left[\alpha(n) \triangleq \phi_{e}(n)\right]$, where '’ stands for: 'equal if one of the algorithms terminates, given the input'.

PRoof: (of theorem 6.2) For each $n \in \mathbb{N}$ we define a recursive real $\beta_{n}$ in $[0,1]$ by defining for each $m \in \mathbb{N}$ the rational number $\beta_{n}(m)$ (a recursive real is given by a Cauchy-sequence of rational numbers 'converging with fixed speed'). For this remember that we fixed a recursive bijection $h$ from $\mathbb{N}^{2}$ to $\mathbb{N}$ in subsection 6.1, and let $n \in \mathbb{N}$ and $m \in \mathbb{N}$. Then (writing ' $\mu k \leq m[A(k)]$ ' to mean 'the least natural number $k$ less than $m$ such that $\left.A(k)^{\prime}\right)$ :

$\beta_{n}(m) \overline{\bar{D}} \begin{cases}\frac{1}{2} & \text { if not } n \in I(\mathbb{N},\{0,1\}) \\ \frac{1}{2} & \text { if } n \in I(\mathbb{N},\{0,1\}) \text { but not } \exists k \leq m[T(n, h(n, 0), k)] \\ \frac{1}{2}-2^{-s} & \text { if } n \in I(\mathbb{N},\{0,1\}) \text { and where } s=\mu k \leq m[T(n, h(n, 0), k)] \text { and } \operatorname{Outc}(s)=1 \\ \frac{1}{2}+2^{-s} & \text { if } n \in I(\mathbb{N},\{0,1\}) \text { and where } s=\mu k \leq m[T(n, h(n, 0), k)] \text { and } \operatorname{Outc}(s)=0\end{cases}$

This definition probably needs some explanation. Informally speaking we are checking whether $n$ is the index of a partial recursive function $\alpha$ from $\mathbb{N}$ to $\{0,1\}$. If so, then $\alpha$ possibly is a total recursive function, in other words $\alpha$ might be a recursive element of $\sigma_{2}$. In that case using $h$ we can see $\alpha$ as a recursive sequence of recursive elements $\left(\alpha^{[n]}\right)_{n \in \mathbb{N}}$ of $\sigma_{2}$, and apply the function $k_{\text {bin }}^{\mathbb{N}}$ to $\alpha$ to find a recursive infinite-real in $[0,1]_{\text {bin }}^{\mathbb{N}}$. For our diagonal argument we are now interested in $\alpha_{n}^{[n]}(0)=\alpha(h(n, 0))$. If at a certain moment $s$ in time the algorithm $\alpha$ finishes computing and yields $\alpha(h(n, 0))$, then we can check whether $\alpha(h(n, 0))=1$ or $\alpha(h(n, 0))=0$. In the first case we are now sure that if $\alpha$ is a recursive element of $\sigma_{2}$, then the $n^{\text {th }}$ coordinate of $k_{\text {bin }}^{\mathbb{N}}(\alpha)$ is a real number greater than or equal to $\frac{1}{2}$ (check!). Likewise in the second case we are sure that if $\alpha$ is a recursive element of $\sigma_{2}$, then the $n^{\text {th }}$ coordinate of $k_{\text {bin }}^{\mathbb{N}}(\alpha)$ is a real number less than or equal to $\frac{1}{2}$.

claim Let $n \in \mathbb{N}$. Suppose $n$ is a recursive index of a recursive element $\alpha$ of $\sigma_{2}$. Then the $n^{\text {th }}$ coordinate of $k_{\text {bin }}^{\mathbb{N}}(\alpha)$ is a real number apart from the real number $\beta_{n}$ defined above.

proof Since $n$ is a recursive index of $\alpha$, we have for all $t \in\{0,1\}$ that $\alpha(h(n, 0))=t$ iff there is an $s \in \mathbb{N}$ such that $T(n, h(n, 0), s)$ and $\operatorname{Outc}(s)=t$.

case $1 \quad t=1$

Then the $n^{\text {th }}$ coordinate of $k_{\text {bin }}^{\mathbb{N}}(\alpha)$ is a real number greater than or equal to $\frac{1}{2}$. On the other hand $\beta_{n} \equiv_{\mathbb{R}} \frac{1}{2}-2^{-s}$ therefore the claim holds.

case $2 \quad t=0$

Then the $n^{\text {th }}$ coordinate of $k_{\text {bin }}^{\mathbb{N}}(\alpha)$ is a real number less than or equal to $\frac{1}{2}$. On the other hand $\beta_{n} \equiv_{\mathbb{R}} \frac{1}{2}+2^{-s}$ therefore the claim holds o

Now let $\beta_{\# \text { bin }}$ be the recursive infinite-real given by $\beta_{\# \text { bin }}=\beta_{0}, \beta_{1}, \ldots$ (or equivalently $\beta_{\# \text { bin }}=\left(\beta_{n}\right)_{n \in \mathbb{N}}$ 
or equivalently $\forall n \in \mathbb{N}\left[\beta_{\# \text { bin }}^{[n]}=\beta_{n}\right]$, whichever notation the reader finds most convenient).

claim The recursive infinite-real $\beta_{\# \text { bin }}$ in $[0,1]^{\mathbb{N}}$ is recursively apart from each recursive binary infinite-real in $[0,1]_{\text {bin }}^{\mathbb{N}}$.

proof Let $\gamma=\gamma_{0}, \gamma_{1}, \ldots$ be a recursive element of $[0,1]_{\text {bin }}^{\mathbb{N}}$. Determine a recursive $\alpha$ in $\sigma_{2}$ such that $\gamma \equiv_{\mathbb{R}^{\mathbb{N}}} k_{\text {bin }}^{\mathbb{N}}(\alpha)$ (see remark 6.1). Determine a recursive index $e$ of $\alpha$. Determine $s \in \mathbb{N}$ such that $T(e, h(e, 0), s)$. By the claim and its proof above $d_{\mathbb{R}}\left(\gamma_{e}, \beta_{e}\right) \geq 2^{-s}$, showing that $\gamma$ is apart from $\beta_{\text {\#bin }}$. Since every function involved is recursive, the claim holds and theorem 6.2 is proved $\circ$

10.2 Now we turn to our remark in 8.5. Following our analysis that the dropping of Cont III is probably wisest, we suggest the following adaptation of the BISH definition of 'locally compact':

DEFinition: Let $(X, d)$ and $\left(Y, d_{Y}\right)$ be metric spaces. $(X, d)$ coincides with $\left(Y, d_{Y}\right)$ iff there is a continuous surjection $h$ from $(X, d)$ to $\left(Y, d_{Y}\right)$ with a continuous inverse $h^{-1}$. Such an $h$ is called a homeomorphism. $\left(Y, d_{Y}\right)$ is called a one-point compact extension of $(X, d)$ iff $\left(Y, d_{Y}\right)$ is compact and there is a $y$ in $Y$ such that $(X, d)$ coincides with $\left(\{z \in Y \mid z \# y\}, d_{Y}\right)$. A metric space $(X, d)$ is locally compact iff $(X, d)$ has a one-point compact extension. $(X, d)$ is called topologically complete iff $(X, d)$ coincides with a complete metric space (then there is a $d$-equivalent $d^{\prime}$ on $X$ such that $\left(X, d^{\prime}\right)$ is complete).

Theorem: Let $(X, d)$ be a locally compact space. Then:

1. For every $x$ in $X$ and every open $U \ni x$ there is a compact neighborhood $W \subseteq U$ of $x$.

2. There is an enumerable neighborhood cover of $(X, d)$ consisting of compact subspaces.

3. There is a $d$-equivalent metric $d^{\prime}$ on $X$ such that in $\left(X, d^{\prime}\right)$ every bounded subset is contained in a compact subspace (therefore $(X, d)$ is topologically complete).

We hold that in all three antipodes the above definition amounts to the desired usual definition of 'locally compact'.

Theorem: (using BDD) For a metric space $(X, d)$ the following statements are equivalent:

1. $(X, d)$ is locally compact

2. For every $x$ in $X$ and every open $U \ni x$ there is a compact neighborhood $W \subseteq U$ of $x$.

3. There is an enumerable neighborhood cover of $(X, d)$ consisting of compact subspaces.

4. There is a $d$-equivalent metric $d^{\prime}$ on $X$ such that in $\left(X, d^{\prime}\right)$ every bounded subset is contained in a compact subspace (therefore $(X, d)$ is topologically complete).

We leave the proof of these theorems as an exercise to the reader, who may consult [Waaldijk1996] and [Bishop\&Bridges1985] (thm. 4.4.9).

10.3 Then we need, for the sake of completeness, to prove a number of statements made in subsection 8.7 and further on. First we show: 
Proposition: $\mathbf{A C}_{10}$ is equivalent to the combination of $\mathbf{C P}, \mathbf{B D D}$ and $\mathbf{A} \mathbf{C}_{00}$.

PROOF: For the implication from right to left see the proof of proposition 8.14. For the implication from left to right the only non-trivial thing to show is that $\mathbf{B D D}$ follows from $\mathbf{A C}_{10}$. Let $B$ be a bar on $\sigma_{\omega}$, let $A=\sigma_{\omega} \times B$. Then we have:

$(\star) \quad \forall \alpha \in \sigma_{\omega} \exists n \in \mathbb{N}[(\alpha, \bar{\alpha}(n)) \in A]$

By $\mathbf{A C}_{10}$ there is a spread-function $\gamma$ from $\sigma_{\omega}$ to $\mathbb{N}$ such that for each $\alpha$ in $\sigma_{\omega}:(\alpha, \gamma(\alpha))$ is in $A$. Therefore $\gamma(\alpha)$ is in $B$ for each $\alpha$ in $\sigma_{\omega}$. Now consider $\alpha$ in $\sigma_{\omega}$. There is a unique $n \in \mathbb{N}$ such that $\gamma(\bar{\alpha}(n))>0$ and we know that $\gamma(\alpha)$ then equals $\gamma(\bar{\alpha}(n))-1$. We hold that $\gamma(\bar{\alpha}(n)) \sqsubseteq \bar{\alpha}(n)$. Otherwise we would have $\bar{\alpha}(n+1) \sqsubseteq \gamma(\bar{\alpha}(n))$, and there would be a $\beta$ in $\sigma_{\omega}$ with $\bar{\beta}(n)=\bar{\alpha}(n)$ yet $\beta(n) \neq \alpha(n)$, whereas by definition of spread-function $\gamma(\beta)=\gamma(\alpha)$ and $\bar{\beta}(n+1) \sqsubseteq \gamma(\bar{\alpha}(n))$, contradiction. So we can take the desired decidable bar to be the following one: $\left\{a \in \overline{\bar{\sigma}}_{\omega} \mid \gamma(a)>0\right\}$

\subsection{LEMMA: BDD follows from $\mathbf{C T}_{11}$ in RUSS.}

PROOF: The proof is a combination of simple building blocks from the literature, which only need little adaptation, and the reasoning in the proof of the lemma above. First one shows, using $\mathbf{C T}_{11}$ : 'every bar on $\sigma_{\omega \mathrm{REC}}$ contains an effective bar on $\sigma_{\omega \mathrm{REC}}$ ', where 'effective' means 'given by a partial recursive function'. Then one proves, using $\mathbf{C T}_{0}$ : every effective bar on $\sigma_{\omega \mathrm{REC}}$ contains a recursively enumerable bar on $\sigma_{\omega \mathrm{REC}}$. This proof can be found in [Ishihara1993]. Finally we must show:

claim Every recursively enumerable bar on $\sigma_{\omega \mathrm{REC}}$ descends from a decidable bar.

proof Let $A=\left\{\phi_{e}(n) \mid n \in \mathbb{N}\right\}$ be a bar on $\sigma_{\omega \mathrm{REC}}$, enumerated by the total recursive function $\phi_{e}$ from $\mathbb{N}$ to $\overline{\bar{\sigma}}_{\omega}$ (for simplicity we view $\overline{\bar{\sigma}}_{\omega}$ as a subset of $\mathbb{N}$ ). We remind the reader that if $T(e, n, k)$ holds for $e, n, k \in \mathbb{N}$, then $n<k$ and $\operatorname{Outc}(k)<k$. Now consider the set $B$ given by: $B=\left\{a \in \overline{\bar{\sigma}}_{\omega} \mid \exists n<\lg (a) \exists b \sqsubset a[T(e, n, \lg (a)) \wedge \operatorname{Outc}(\lg (a))=b]\right\}$. Since $A$ is a bar, $B$ is a decidable bar such that $A$ descends from $B$ ○

10.5 We also need to derive $\mathbf{C P}_{\text {Baire }}$ from CP. For this we repeat a theorem from [Waaldijk1996], which is proved therein using only $\mathbf{C P}$.

Theorem: $\left(\mathbf{C P}_{\mathrm{cm}}\right)$ let $(X, d)$ be a complete metric space. Let $A$ be a subset of $X \times \mathbb{N}$ such that:

(i) $\forall x \in X \exists n \in \mathbb{N}[(x, n) \in A]$

(ii) $\forall x, y \in X \forall n \in \mathbb{N}[(x \equiv y \wedge(x, n) \in A) \rightarrow(y, n) \in A]$

Then: $\forall x \in X \exists n, m \in \mathbb{N} \forall y \in X\left[d(x, y)<2^{-m} \rightarrow(y, n) \in A\right]$.

From this theorem it is easy to derive $\mathbf{C P}_{\text {Baire, }}$ we leave this as an exercise.

10.6 REMARK: As a last remark we offer a weak version of FT which is true in BISH if one accepts the definition of genetic bars and PGI. The proof is a simple application of PGI. Notice that FT follows trivially from $\mathbf{F T}_{0}$ and Brouwer's Thesis. 
PROPOSITION: $\left(\mathbf{F T}_{0}\right)$ Every genetic bar on $\sigma_{2}$ contains a finite bar.

\section{BiBliogRAPHY}

[Bishop1967]

[Bishop\&Bridges1985]

[Bishop1985]

[Bridges1979]

[Bridges\&Richman1987]

[Brouwer1907]

[Brouwer1922]

[Brouwer1975]

[vanDalen1999]

[DeFinetti1972]

[DeFinetti1974]

[Dugundji1951]

[Earman1986]

[FoM2001]

[Gielen et al. 1981]

[Heyting1956]

[Hill1996]

[Ishihara1993]
E. Bishop, Foundations of Constructive Analysis, McGraw-Hill, New York, 1967.

E. Bishop and D.S. Bridges, Constructive Analysis, Springer, Berlin, 1985.

E. Bishop, Schizophrenia in Contemporary Mathematics, Erret Bishop: Reflections on Him and His Research (ed. Rosenblatt), pp. 1-32; AMS, Providence, Rhode Island, 1985.

D.S. Bridges, Constructive Functional Analysis, Pitman, London, 1979.

D.S. Bridges and F.Richman, Varieties of constructive mathematics, London Math. Soc. Lecture Notes no. 93, Cambridge University Press, 1987.

L.E.J. Brouwer, Over de Grondslagen der Wiskunde, PhD thesis, Universiteit van Amsterdam, 1907.

L.E.J. Brouwer, Besitzt jede reelle Zahl eine Dezimalbruch-Entwickelung?, Math. Annalen 83, 201-210, 1922.

L.E.J. Brouwer, Collected works, (vol. I, II) North-Holland, Amsterdam, 1975.

D. van Dalen, Mystic, Geometer and Intuitionist - The Life of L.E.J. Brouwer, vol. 1 The Dawning Revolution, Clarendon Press, Oxford, 1999.

B. De Finetti, Probability, Induction and Statistics, Wiley, New York, 1972.

B. De Finetti, Theory of Probability - A critical introductory treatment, (vol. I, II) Wiley, New York, 1974.

J. Dugundji, An extension of Tietze's theorem, Pacific Journal of Mathematics 1, 353-367, 1951.

J. Earman, A Primer on Determinism, D. Reidel Publ., Dordrecht, 1986.

S. Simpson (ed.), Foundations of Mathematics forum, http://www.cs.nyu.edu/pipermail/fom/1998-November and /2001January, 1998-2001.

W. Gielen, H. de Swart and W.H.M. Veldman, The Continuum Hypothesis in Intuitionism, Journal of Symbolic Logic 46 (1), 121-136, 1981.

A. Heyting, Intuitionism - an Introduction, North-Holland, Amsterdam, 1956 (3rd rev. ed. 1971).

T. Hill, A statistical derivation of the significant-digit law, Statistical Science 10, 354-363, 1996.

H. Ishihara, Markov's Principle, Church's thesis and Lindelöf's theorem, Indag. Mathem., N.S., 4 (3), 321-325, 1993. 
[Ishihara1994]

[Johnson1987]

[Kleene1952]

[Kleene\&Vesley1965]

[Kushner1985]

[Michael1956]

[Troelstra\&vanDalen1988]

[Veldman1981]

[Veldman1985]

[Waaldijk1996]
H. Ishihara, A constructive version of Banach's inverse mapping theorem, New Zealand Journal of Mathematics, vol. 23, 71-75, 1994.

D.M. Johnson, L.E.J. Brouwer's coming of age as a topologist, MAA Studies in Mathematics, vol. 26, Studies in the History of Mathematics (ed. E.R. Phillips), 61-97, 1987.

S.C. Kleene, Introduction to metamathematics, North-Holland, Amsterdam, 1952.

S.C. Kleene and R.E. Vesley, The Foundations of Intuitionistic Mathematics - especially in relation to the theory of recursive functions, NorthHolland, Amsterdam, 1965.

B.A. Kushner, Lectures on Constructive Mathematical Analysis, American Mathematical Society, Providence, R.I., 1985.

E. Michael, Continuous selections (I), Annals of Mathematics 63, 361-382, 1956.

A.S. Troelstra and D. van Dalen, Constructivism in Mathematics, (vol. I, II) North-Holland, Amsterdam, 1988.

W.H.M. Veldman, Investigations in intuitionistic hierarchy theory, $\mathrm{PhD}$ thesis, University of Nijmegen, 1981.

W.H.M. Veldman, Intuïtionistische wiskunde, (lecture notes in Dutch) University of Nijmegen, 1985.

F.A. Waaldijk, modern intuitionistic topology, $\mathrm{PhD}$ thesis, University of Nijmegen, 1996. 